\title{
Abstracts from the 2019 Annual Scientific Meeting of the British and Irish Hypertension Society (BIHS)
}

Published online: 16 September 2019

(c) The Author(s), under exclusive licence to Springer Nature Limited 2019

Edgbaston Park Hotel and Conference Centre, Birmingham, 16-18 September 2019

Conference Organizer: In Conference Ltd on behalf of BIHS. https://bihsoc.org/events/annual-scientific-meeting/

All content was reviewed and approved by members of the British and Irish Hypertension Society, which held full responsibility for the abstract selections.

Sponsorship: Funding for the publication of this supplement was provided by the British and Irish Hypertension Society for the advancement of knowledge and dissemination of information concerning the pathophysiology, epidemiology, detection, investigation and treatment of arterial hypertension and related vascular diseases.

Presenting authors have asterisks in the contributor lists

O-01 Blood pressure and capillary microcirculation in term and preterm infants of mothers with hypertensive disorders of pregnancy

Muti Goloba ${ }^{* 1,2}$, Rajendra Raghuraman ${ }^{1}$, Uzma Khan ${ }^{1}$, Nansi Botros ${ }^{1}$, Monique Klein ${ }^{1}$, Amelia Brown ${ }^{1}$, Donovan Duffy $^{2}$, Nick Anim-Nyame ${ }^{3}$, Duolao Wang ${ }^{4}$, Isaac Manyonda $^{2}$, Tarek Antonios ${ }^{1,2}$

${ }^{1}$ St George's University of London, London, United Kingdom, ${ }^{2}$ St George's University Hospitals NHS Foundation Trust, London, United Kingdom, ${ }^{3}$ Kingston Hospital NHS Foundation Trust, London, United Kingdom, ${ }^{4}$ Liverpool School of Tropical Medicine, Liverpool, United Kingdom

Introduction: Offspring of mothers with hypertensive disorders of pregnancy (HDP) are at increased future risk of hypertension, cardiovascular disease and stroke. The pathophysiological mechanisms for this association are not well understood, but microvascular abnormalities and in particular capillary rarefaction (CR) have been implicated. In a previous pilot study, we found that infants born preterm to HDP mothers have significant CR at birth.

Methods: We studied 111 infants born to mothers with an HDP (90 born at term (T-HDP) and 21 born pre-term (PT-HDP) and compared them to 278 normal birth weight infants born at term to normotensive mothers (control group). We used intravital capillary microscopy to measure basal i.e. functional (BCD) and maximal i.e. structural (MCD) capillary densities and BP at birth, 3 months, 6 months and 12 months. We report here BP and capillary density results at birth.

Results: PT-HDP infants had a significantly higher BCD (mean difference 12.62 cap/field; $p=0.001$ ) and (mean difference 17.26 cap/field; $p<0.001$ ) compared to T-HDP infants and controls respectively. T-HDP infants also had a significantly higher BCD (mean difference 4.64, cap/field; $p=0.012$ ) compared to control infants). PT-HDP infants also had higher MCD (mean difference 10.53 cap/field; $p=0.003$ ) and (mean difference 13.67 cap/field; $p<0.001$ ) respectively compared to T-HDP and control infants. Systolic BP was significantly higher in PT-HDP infants compared to control infants (mean difference $7.19 \mathrm{mmHg}$; $p=0.032$ ). There were no significant differences in diastolic BP.

Conclusions: PT-HDP infants have a significantly higher systolic BP at birth compared to infants of normotensive mothers. Furthermore, PT-HDP have, contrary to our pilot study, a significantly higher BCD and MCD compared to NBW infants of normotensive mothers. Further follow-up studies of these infants are required to investigate the crucial role of microcirculatory abnormalities in the pathogenesis of future hypertension. 
Disclosure: The study was funded by the BHF (PG/13/ 87/30550)

\section{O-02 Haemodynamic determinants of essential hyper- tension in children}

\section{Ye $\mathrm{Li}^{* 1}$, Haotian $\mathrm{Gu}^{1}$, Manish Sinha ${ }^{1,2}$, Phil Chowienczyk $^{1}$}

${ }^{1}$ King's College London, London, United Kingdom, ${ }^{2}$ Evelina London Children's Hospital, London, United Kingdom

Introduction: Haemodynamic determinants of hypertension in young adults include increased stroke volume and arterial stiffness [1]. The aim of the present study was to examine the contribution of left ventricular ejection, arterial stiffness and systemic vascular resistance to hypertension in children with essential hypertension.

Methods: A total of 81 children aged 7 to 18 years (mean age 14 years) including 31 with hypertension and 50 age matched normotensive children were recruited from the paediatric hypertension clinic and local community. 18 (58.1\%) hypertensive subjects were taking antihypertensive medication. Non-invasive aortic flow velocity and central blood pressure were measured by Doppler sonography and carotid tonometry system respectively. Aortic pulse wave velocity was calculated using the sum of squares method [2].

Results: Compared with normotensive subjects, those with essential hypertension had significantly higher central pulse pressure ( $43 \pm 15$ vs. $29 \pm 8 \mathrm{mmHg}$, mean \pm SD), heart rate ( $81 \pm 15$ vs. $73 \pm 11 \mathrm{bpm})$, and cardiac output (5.4 \pm 2.0 vs. $4.3 \pm 1.2 \mathrm{~L} / \mathrm{min}), P<0.01$ for all comparisons. There was a trend toward increased stroke volume in hypertensive subjects, but not significantly different from normotensive subjects. However, there were no differences in augmentation pressure, reflection index or systemic vascular resistance between the two groups. The increase in central pulse pressure was explained by an altered pattern of ventricular ejection (initial flow increased from 1.13 to $1.26 \mathrm{~m} / \mathrm{s}, P=$ $0.008)$ and increase in aortic pulse wave velocity from 2.5 to $3.4 \mathrm{~m} / \mathrm{s}(P=0.001)$.

Conclusions: These results suggest that increase in central pulse pressure in hypertensive compared to normotensive children is explained by an increase in arterial stiffening and altered pattern of ventricular ejection.

Disclosure: None

References:

1. McEniery CM, Yasmin, Wallace S, Maki-Petaja K, McDonnell B, Sharman JE, et al. Increased stroke volume and aortic stiffness contribute to isolated systolic hypertension in young adults. Hypertension. 2005;46(1):221-6.

2. Davies JE, Whinnett ZI, Francis DP, Willson K, Foale RA, Malik IS, et al. Use of simultaneous pressure and velocity measurements to estimate arterial wave speed at a single site in humans. Am J Physiol Heart Circ physiol. 2006;290(2): H878-85.

\section{O-03 Omitted}

O-04 Central pressure in healthy adults stratified by MTHFR 677 genotype

Martina Rooney ${ }^{* 1}$, Catherine Hughes ${ }^{1}$, Michelle Clements $^{I}$, Sean Strain ${ }^{1}$, Helene McNulty ${ }^{1}$, Mary Ward ${ }^{1}$

${ }^{1}$ Ulster University, Coleraine, United Kingdom

Introduction: Hypertension is the leading risk factor for cardiovascular disease (CVD). A common C677T polymorphism in the folate metabolising enzyme, methylenetetrahydrofolate reductase (MTHFR) is associated with an increased risk of hypertension by $24-87 \%$. Randomised controlled trial evidence from our Centre has demonstrated that riboflavin, the MTHFR cofactor, can lower blood pressure (BP) by $5-13 \mathrm{mmHg}$, an effect confined to homozygous individuals (TT genotype). To date, our trials have focused on peripheral BP; however, central pressure, which is more closely correlated with CVD risk, may offer further insight into the role of this gene-nutrient interaction in BP. The aim of this study was to investigate both peripheral and central BP in apparently healthy adults, stratified by MTHFR genotype.

Methods: Adults aged 18-60 years recruited from workplaces across Northern Ireland were screened for MTHFR genotype. Individuals with TT genotype were ageand sex-matched to $\mathrm{CC} / \mathrm{CT}$ controls. Brachial BP and central BP (SphygmoCor ${ }^{\circledR}$, AtCor Medical, Australia) were measured. A blood sample and lifestyle information were also obtained.

Results: Preliminary results ( $n$ 409) confirm elevated BP in individuals with the MTHFR 677TT genotype compared to $\mathrm{CC} / \mathrm{CT}$ controls (systolic BP $134.7 \pm 13.8 \mathrm{mmHg}$ vs. $129.7 \pm 12.4 \mathrm{mmHg}, \quad P<0.001 ; \quad$ diastolic BP $81.6 \pm$ $9.5 \mathrm{mmHg}$ vs $79.7 \mathrm{mmHg} \pm 8.9 \mathrm{mmHg}, P=0.023)$. The MTHFR 677TT genotype group had significantly higher central systolic BP $(119.4 \pm 11.8$ vs $116.7 \pm 10.9 \mathrm{mmHg}$, $P=0.018)$, central pulse pressure $(P=0.006)$ and central mean pressure $(P=0.011)$ compared to the non-TT group. No significant differences for central diastolic BP, pulse pressure amplification, pulse pressure ratio and augmentation index were observed. 
Conclusions: This study confirms the phenotype of elevated BP in individuals with the MTHFR 677TT genotype. For the first time elevated central systolic BP, pulse pressure, and central mean pressure in these genetically atrisk adults is demonstrated. The effect of riboflavin, the MTHFR cofactor, on measures of central pressure warrants further investigation.

Disclosure: None

\section{O-05 Omitted}

O-06 Translating signals from genome-wide association studies into biological mechanisms of hypertension through kidney-omics

Xiao Jiang ${ }^{*}$, James Eales ${ }^{1}$, Xiaoguang $\mathrm{Xu}^{1}$, Artur Akbarov ${ }^{I}$, Sanjeev Pramanik ${ }^{I}$, Pawel Bogdanski, Wojciech Wystrychowski, Joanna Zywiec ${ }^{4}$, Ewa Zukowska-Szczechowska ${ }^{5}$, Adrian Woolf ${ }^{\prime}$, Nilesh J. Samani $^{2,6}$, Fadi J. Charchar ${ }^{2,7}$, Maciej Tomaszewski ${ }^{1,8}$

${ }^{1}$ The University of Manchester, Manchester, United Kingdom, ${ }^{2}$ University of Leicester, Leicester, United Kingdom, ${ }^{3}$ Karol Marcinkowski University of Medical Sciences, Poznan, Poland, ${ }^{4}$ Medical University of Silesia, Katowice, Poland, ${ }^{5}$ Silesian Medical College, Katowice, Poland, ${ }^{6}$ Glenfield Hospital, Leicester, United Kingdom, ${ }^{7}$ Federation University Australia, Ballarat, VIC, Australia, ${ }^{8}$ Manchester Academic Health Science Centre, Manchester, United Kingdom

Introduction: Genome-wide association studies (GWAS) have identified over 800 single nucleotide polymorphisms (SNPs) associated with blood pressure (BP). The biological mechanisms of their associations with BP are not clear. We hypothsised that a significant proportion of these variants operate through effects on gene expression (e), alternative splicing(s) and methylation(m) in the human kidney.

Methods: We collected 430 human kidneys from 5 studies. Transcriptome profiling was performed by nextgeneration RNA-sequencing. Quantification of splicing ratios was performed using LeafCutter. DNA methylation levels were measured using Infinium Human Methylation 450 array and EPIC array. Genotyping was collected by Infinium Human CoreExome-24 BeadChip array or Affymetrix SNP 6.0 array. We conducted cis-quantitative trait locus (QTL) analyses on the three layers of kidney omics followed by colocalisation and Mendelian randomisation (MR) analyses.

Results: We discovered a total of 18,201 renal genes, 50,982 intron excision clusters (IECs) and 374,826 CpG sites in human kidney. After correction for multiple testing, we identified 7348 genes (kidney eGenes), 6799 IECs (kidney sIECs) and 34,913 CpG sites (kidney $\mathrm{mCpG}$ ) from cis-QTL analyses. We uncovered that 58\% BP GWAS loci have at least one functional (expression, splicing or methylation) signature. In $31 \%$ of these BP GWAS loci, the expression, splicing or methylation target share the same causal genetic variant with BP. MR further confirmed 98 kidney targets as causally related to BP.

Conclusions: We uncovered the identity of renal genes and molecules operating as the regulators of BP. These genes are excellent targets for the development of new therapeutic strategies.

Disclosure: None

O-07 Vasodilator prostaglandins make an important contribution to forearm vascular responses to mental stress in young South Asians and may limit their risk of cardiovascular disease

\section{Janice Marshall, Abimbola Aiku ${ }^{* 1,2}$, Una Martin ${ }^{1}$}

${ }^{1}$ University of Birmingham, Birmingham, United Kingdom, ${ }^{2}$ University of Ibadan, Nigeria

Introduction: South Asians (SAs) have greater risk of cardiovascular disease (CVD) than White Europeans (WEs) [1]. Accordingly, we reported that young SA men show blunted reactive hyperaemia and forearm vasodilator responses to mental stress relative to WE men [2]. Whether these responses are blunted in SA women and whether prostaglandins (PGs) contribute to either response in SAs is unknown.

Methods: In Group 1 (9 WE, 9 SA women; 18-26 years), we recorded mean arterial pressure (MAP) and forearm blood flow (FBF) during reactive hyperaemia $(\mathrm{RH})$ and 5 sound stress stimuli (S1-S5; $100 \mathrm{~dB}, 2 \mathrm{KHz}, 30$ s at 5-10 min intervals): forearm vascular conductance (FVC); FBF/MAP). In Group 2 (14 SAs; 6 men/9 women; 19 WEs:10/9), this protocol was performed without and after cyclooxygenase (COX) inhibition (aspirin, $600 \mathrm{mg}$ p.o).

Results: In Group 1, RH was similar in WE and SA women $(\triangle \mathrm{FVC}:+0.28 \pm 0.03$ vs $+0.32 \pm 0.02$ conductance units (CU)). However, S1-S5 evoked forearm vasodilatation in WE women, but mean vasoconstriction in SA women $(\triangle \mathrm{FVC}$ : $+0.005+0.003$ vs $-0.009 \pm 0.004 \mathrm{CU}$ ), more individual SA women showing forearm vasoconstriction (63.6 vs 33.3\%). In Group 2, SA men and women who showed forearm vasoconstriction (vasoconstrictors) to S1-S5 showed blunted RH relative to vasodilators $(+0.45 \pm 0.05$ vs $+0.31 \pm 0.02 \mathrm{CU}, p=0.03)$. Further, COX inhibition had no effect on RH, or SS1-5 on 
vasoconstrictors to SS1-5 (RH: $0.31 \pm 0.02$ vs $0.38 \pm$ $0.02 \mathrm{CU}$; SS1-5: $-0.012 \pm 0.005$ vs $-0.033 \pm 0.005 \mathrm{CU})$, but attenuated RH and SS1-5 in vasodilators to SS1-5 (RH: $0.45 \pm 0.05$ to $0.30 \pm 0.03 \mathrm{CU}, p=0.05$; S1-S5: $0.011 \pm$ 0.005 vs $0.003 \pm 0.002 \mathrm{CU}, p=0.004$ ).

Conclusions: Reactive hyperaemia, which is endothelium-dependent, is of similar magnitude in young SA and WE women. However, SA women and men show blunted forearm vasodilatation or in the majority of individuals, predominating vasoconstriction to repeated environmental stress. Those SAs who show vasodilatation may be protected by vasodilator PGs, but those who show vasoconstriction may be particularly at risk of stressinduced CVD.

Disclosure: None

References:

1. Gupta M, Singh N, Verma S. Circulation 2006;113: 924-29.

2. Ormshaw NG, Junejo RT, Marshall JM. J Appl Physiol. 2018;118:979-88.

O-08 The effects of indapamide on glycosaminoglycanmediated non-osmotic skin sodium storage in humans

Kathleen Connolly ${ }^{* 1}$, Viknesh Selvarajah, James Goodman ${ }^{I}$, Spoorthy Burli, Douglas Maslin ${ }^{I}$, Michalis Kostapanos ${ }^{1}$, Lucy Yang ${ }^{2}$, Carmel McEniery ${ }^{1}$, Ian Wilkinson ${ }^{I}$

${ }^{1}$ University of Cambridge, Cambridge, United Kingdom, ${ }^{2}$ University College of London Hospital, London, United Kingdom

Introduction: Animal data suggest that the skin may protect against the adverse haemodynamic effects of dietary salt by storing sodium in an osmotically inactive form, bound to glycosaminoglycans in the extracellular matrix. However, to date this novel pathway has not been comprehensively studied in humans.

Methods: 85 healthy volunteers (37 males, 48 female) under 50 years were recruited into a double-blind parallel study design. Participants underwent a one-week run-in period to standardise dietary salt intake before random allocation to either lactose placebo or $2.5 \mathrm{mg}$ indapamide diuretic daily for eight days.

Total body water (TBW) quantification, haemodynamic measurements, and skin biopsies were performed before and after treatment. Peripheral blood pressures were measured after $5 \mathrm{~min}$ seated rest. Pulse wave velocity (PWV) was measured using SphygmoCor, and stroke volume (SV) and cardiac output (CO) were measured by inert gas rebreathing.
$5 \mathrm{~mm}$ skin biopsies were taken from the lower back using sodium-free lignocaine. Biopsies were freeze-dried to establish water content, whilst sodium content was quantified using inductively coupled plasma-optical emission spectroscopy. Glycsosaminoglycan content was assessed by qPCR. Data were analysed using repeated measures ANOVA.

Results: Following indapamide treatment, skin sodium fell by $10 \%(p=0.004)$ with no reduction in skin water content, compared to placebo. There were no significant differences in blood pressure or PWV between groups. $\mathrm{TBW}, \mathrm{SV}$, and $\mathrm{CO}$ were significantly reduced by indapamide compared to placebo $(p<0.001, p=0.007, p=0.009$, respectively), whilst systemic vascular resistance increased $(p<0.001)$. mRNA expression of glycomsaminoglycan synthetic enzymes were reduced $(p<0.05)$.

Conclusions: A diuretic-induced reduction in skin sodium, without commensurate water loss, suggests release of sodium from osmotically inactive skin stores, while the corresponding drop in glycosaminoglycan synthetic enzyme expression suggests these stores are regulated by glycosaminoglycans in humans. This is the first comprehensive evidence of glycosaminoglycan-regulated non-osmotic skin sodium storage in humans.

Disclosure: None

O-09 Effect of a Reduction in glomerular filtration rate after NEphrectomy on arterial STiffness and central haemodynamics: the EARNEST study

\section{Anna Price ${ }^{* 1}$, George Greenhall, Charles Ferro ${ }^{1}$, Ian Wilkinson $^{1}$, Laurie Tomlinson ${ }^{1}$, Jonathan Townend ${ }^{1}$}

${ }^{1}$ On behalf of the EARNEST study investigators, United Kingdom

Introduction: Chronic kidney disease (CKD) is a leading risk factor for cardiovascular events and mortality [1]. Hypertension and arterial stiffness are both considered fundamental intermediaries to cardiac disease in this population yet existing hypertension is widespread in CKD [2]. It is uncertain whether arterial stiffness is related to a reduction in glomerular filtration rate or the many co-morbid conditions including hypertension which accompany CKD. EARNEST was a multi-centre prospective controlled study designed to investigate the haemodynamic effects of an isolated reduction in kidney function due to uninephrectomy.

Methods: Living kidney donors and healthy controls were recruited from seven UK centres. Inclusion and exclusion criteria for donors and controls were set in accordance with national living kidney donor criteria. Participants underwent ambulatory blood pressure (using the 
Mobilo-O-Graph ${ }^{\circledR}$ ) and assessment of arterial stiffness (using the SphygmaCor) at baseline and 12 months.

Results: A total of 469 participants were recruited, of which 306 (138 controls and 168 donors) attended follow up. There were no differences between donors and controls in either ambulatory day systolic blood pressure (123.78 \pm 9.77 vs $122.90 \pm 11.66 \mathrm{mmHg}, p=0.534$ ) or ambulatory day diastolic blood pressure $(78.90 \pm 7.97$ vs $77.89 \pm$ $8.87 \mathrm{mmHg}, p=0.358$ ) at 12 months. There were also no significant differences in ambulatory night blood pressures.

Mean change in pulse wave velocity (PWV) was not significantly different in donors compared to controls $(+0.299$ vs $+0.122 \mathrm{~m} / \mathrm{s}, p=0.120)$. In a multivariable model, kidney donation was not independently associated with a change in PWV ( $\beta$ coefficient 0.17 (confidence interval $-0.049-0.404), p=0.126$ ).

Conclusions: At 12 months after nephrectomy, there was no significant change in ambulatory peripheral blood pressure or pulse wave velocity. Living kidney donors can be reassured that at least at one year after donation there is no evidence of a rise in peripheral blood pressure or pulse wave velocity.

Disclosure: None

\section{References:}

1. Dalrymple LS, Katz R, Kestenbaum B, Shlipak MG, Sarnak MJ, Stehman-Breen C. et al. Chronic kidney disease and the risk of end-stage renal disease versus death. J Gen Intern Med. 2011;26:379-85.

2. Coresh J, Selvin E, Stevens LA, et al. Prevalence of chronic kidney disease in the United States. JAMA. 2007;298:2038-47.

\section{O-10 Omitted}

0-11 A novel non-invasive cuff-less optoelectronic sensor to measure blood pressure: comparison against intra-arterial measurement

\section{Melvin Lobo ${ }^{* 1,2}$, Arthur Tucker ${ }^{1,2}$, Maudrian Burton ${ }^{2}$, Andrea McDonnell', Nita Shah ${ }^{3}$, Sandeep Shah ${ }^{3}$, Benjamin O'Brien $^{1,2}$}

${ }^{1}$ Queen Mary University London, London, United Kingdom, ${ }^{2}$ Barts Health NHS Trust, London, United Kingdom, ${ }^{3}$ Tarilian Laser Technologies, Welwyn Garden City, United Kingdom,

Introduction: A newly developed non-invasive cuff-less BP sensor enables high fidelity direct beat-to-beat measurement of BP in digital format using patented optoelectronic methods without need for calibration, compression or energy entering the body. The sensor's main components comprise a light emitting diode (LED) utilising a semiconductor light source, a waveguide, a photodiode and a flexible button which is placed on the skin over the radial artery all housed within a strap. We evaluated the accuracy of this device compared to an intra-arterial BP catheter according to the ISO-81060-2:2013 standard.

Methods: Patients were recruited who were indicated to receive an invasive radial arterial $\mathrm{BP}$ catheter as a standard of clinical care. Non-invasive BP was measured with the deviceunder-test which was applied in the post-operative period to the contralateral wrist to the radial arterial line. The reference $\mathrm{BP}$ ranges were determined from the recording of the invasive $\mathrm{BP}$ for a duration of at least $30 \mathrm{~s}$ that included the period of the determination of the device-under-test for which $10 \mathrm{BP}$ determinations per patient were recorded.

Results: In total 12 adult men and 6 women with mean age of 59 years, BMI $<35 \mathrm{kgm} 2$ and in sinus rhythm were studied. For the 180 separate pairs of readings, the mean values of the errors of the determinations between the indwelling arterial line and the optoelectronic device-under-test were:

Systolic BP: $-0.22 \mathrm{mmHg}$ (SD 2.76), ISO standard requires $\pm 5 \mathrm{mmHg}, \mathrm{SD}<8$

Diastolic BP: $+3.91 \mathrm{mmHg}$ (SD 5.04), ISO standard requires $\pm 5 \mathrm{mmHg}, \mathrm{SD}<8$

Conclusions: This is the first time a static optoelectronic $\mathrm{BP}$ sensor has been compared to the intra-arterial BP gold standard. The results obtained for both systolic and diastolic pressures meets the criterion of the ISO Standard and so the device-under-test can be recommended for blood pressure measurement in an adult population.

Disclosure: MDL is a consultant to TLT, Medtronic, ReCor Medical, Ablative Solutions, Vascular Dynamics, and ROX Medical. He has received speaker fees from CVRx. AT is a consultant to TLT, Barchester Group Ltd, Edoximed Ltd, Sky Medical Technology Ltd, FirstKind Ltd And Seahorse Group. NS and SS are founders and shareholders of TLT. BO'B is a consultant to TLT, Correvio Pharma and Medtronic. $\mathrm{MB}$ and $\mathrm{AM}$ have nothing to disclose.

0-12 Diastolic dysfunction is associated with prolonged ventricular contraction in children with essential hypertension

Haotian $\mathrm{Gu}^{* 1}$, Ye Lit, Phil Chowienczyk ${ }^{1}$, Manish Sinha ${ }^{2}$

${ }^{1}$ King's College London, London, United Kingdom, ${ }^{2}$ Evelina London Children's Hospital, London, United Kingdom

Introduction: In adults with hypertension, diastolic dysfunction is associated with prolonged systolic contraction 
[1]. We investigated whether this is also the case in children with hypertension.

Methods: Transthoracic echocardiography and carotid tonometry were performed in 31 children with essential hypertension and 50 age matched normotensive subjects. Left ventricular (LV) cavity and epicardial volumes was obtained from 4-chamber view using Tomtec wall tracking analysis. The ratio of mitral valve Doppler early flow (E) and tissue Doppler mitral annulus movement (e') was used (E/e') as a measure of diastolic function. Time-varying ejection phase myocardial wall stress (MWS) was calculated from LV volumes and central aortic pressure derived from carotid tonometry [2]. Time to onset of relaxation (TOR) was defined as time to peak MWS over ejection time. Pulse wave velocity (PWV) was calculated from aortic arch to abdominal aorta using pulsed Doppler from echocardiography.

Results: Hypertensive children were of similar age and BMI compared to normotensive children. Hypertensive children had increased LV mass index $(p=0.001)$ and relative wall thickness $(p<0.001)$ suggestive of concentric remodelling. There was no difference in LV systolic measures including ejection fraction and global longitudinal strain between hypertensives and normotensives. However, hypertensives had higher E/e' ratio $(6.4 \pm 1.1$ vs $5.5 \pm 1.0$, $p<0.001)$, PWV $(5.1 \pm 1.5$ vs $4.3 \pm 0.9 \mathrm{~m} / \mathrm{s}, p=0.023)$ and prolonged TOR $(32.5 \pm 9.2$ vs $28.8 \pm 5.6 \%, p=0.027)$ and elevated mean MWS $(409.7 \pm 93.1$ vs $336.6 \pm 86.6$ $\left.\mathrm{kdynes} / \mathrm{cm}^{2}, p=0.001\right)$ than normotensives. TOR was positively associated with E/e' ratio $(\beta=0.277, p=0.01)$ with or without adjustment for age, sex, BMI, mean arterial pressure, HR, left ventricular mass and antihypertensive treatments.

Conclusions: In children with essential hypertension and normal systolic function, pre-clinical diastolic dysfunction is associated with prolonged systolic contraction.

Disclosure: None

References:

1. Gu H, Li Y, Fok H, Simpson J, Kentish JC, Shah AM, Chowienczyk PJ. Reduced first-phase ejection fraction and sustained myocardial wall stress in hypertensive patients with diastolic dysfunction: a manifestation of impaired shortening deactivation that links systolic to diastolic dysfunction and preserves systolic ejection fraction. Hypertension. 2017;69:633-40.

2. Chirinos JA, Segers P, Gupta AK et al. Time-varying myocardial stress and systolic pressure-stress relationship: role in myocardial-arterial coupling in hypertension. Circulation. 2009;119:2798-807.

O-13 Mechanisms underlying obesity-related hypertension: an experimental weight gain study in humans
Jessica Middlemiss ${ }^{1}$, Kaisa Maki-Petaja ${ }^{1}$, Carmel McEniery $^{* 1}$

${ }^{1}$ University of Cambridge, Cambridge, United Kingdom

Introduction: The association between body size and blood pressure (BP) is well recognised. However, not all overweight individuals have elevated BP suggesting that important adaptive mechanisms are present in some. Indeed, previous findings suggest that the ability to modulate peripheral vascular resistance (PVR) in response to elevated cardiac output $(\mathrm{CO})$ is important in determining $\mathrm{BP}$ in young overweight adults. In preparation for examining this hypothesis, we have undertaken an exploratory study of experimental weight gain in healthy young adults.

Methods: Sixteen healthy non-obese males were recruited (mean \pm SD age $29 \pm 6$ years). All participants were overfed approximately $1000 \mathrm{kcal} / \mathrm{day}$, alongside their normal diet, for 6-8 weeks, until a $5 \mathrm{~kg}$ weight gain was achieved and held stable for at least 1 week. Detailed anthropometric characteristics, BP, CO and PVR were assessed at baseline and at completion of the overfeeding intervention.

Results: Overfeeding significantly increased body weight $(6.11 \pm 3.08 \mathrm{~kg}, \quad P<0.001)$, lean mass $(3.61 \pm$ $3.40 \mathrm{~kg}, \quad P=0.001)$ and fat mass $(2.64 \pm 2.89 \mathrm{~kg}$, $P=0.002)$. The increases in systolic BP $(2 \pm 9 \mathrm{mmHg})$ and diastolic BP $(2 \pm 6 \mathrm{mmHg})$ were not significant $(P<0.2$ for both) and the increase in heart rate was of borderline significance $(5 \pm 10 \mathrm{bpm}, P=0.055)$. There was a significant increase in $\mathrm{CO}(0.81 \pm 1.35 \mathrm{~L} / \mathrm{min}, P=0.031)$, which was accompanied by a decrease in PVR $\left(129 \pm 205\right.$ dynes.s.cm ${ }^{5}$, $P=0.024)$. Dichotomising individuals based on a systolic BP response to weight gain $\geq 5 \mathrm{mmHg}$ (BP responders) revealed small differences vs non-responders in the change in $\mathrm{CO}(+1.34 \pm 0.99 \mathrm{~L} / \mathrm{min}$ vs $+0.4 \pm 1.50 \mathrm{~L} / \mathrm{min})$ and PVR $\left(-111 \pm 142\right.$ dynes.s.cm ${ }^{5}$ vs $-143 \pm 251$ dynes.s.cm $\left.{ }^{5}\right)$, although neither was statistically significant.

Conclusions: Modest weight gain in healthy, non-obese men is associated with increases in lean and fat mass. A compensatory reduction in PVR in response to elevated cardiac output is likely to explain the lack of change in BP. Examining 'BP responders' versus 'BP non responders' is likely to provide useful insights into obesity-associated hypertension in a larger trial.

Disclosure: None

\section{O-14 Omitted}

O-15 A novel 4 Pillar model of lifestyle intervention reduces blood pressure in cardiovascular disease and maintains this after 1-year 
Sam Olden ${ }^{*}$, Gavinder Kandola ${ }^{1}$

${ }^{1} \mathrm{CP}+\mathrm{R}$, London, United Kingdom

Introduction: Every $10 \mathrm{mmHg}$ reduction in systolic blood pressure (SBP) reduces mortality rate risk by $13 \%$ [1]. This study presents a novel method of reducing blood pressure through bespoke lifestyle interventions.

Methods: 386 participants $(326 \mathrm{M}, 60 \mathrm{~F}$; aged 58.4 years) with a history of cardiac disease and 47 participants (26 M $21 \mathrm{~F}$; aged 51.0 years) with no history of clinical disease were included. All individuals were on optimal medical therapy prior to commencement. All participants completed a 12-week cardiac rehabilitation programme and could opt to continue for 48 weeks. This involved twiceweekly supervised strength training sessions and steadystate aerobic training, step-count monitoring and nutritional guidance. Participants underwent pre and post measures of SBP.

Results: Significant reductions in SBP were found at 12weeks and continued to 48 weeks. Sub-groups showed the following reductions from baseline: PCI $(n=90)$ $-7.2 \mathrm{mmHg}$ at 12 weeks, $-8.3 \mathrm{mmHg}$ at 48 weeks; CABG $(n=33)-8.4 \mathrm{mmHg}$ at 12 weeks, $-12.9 \mathrm{mmHg}$ at 48 weeks; HTN $(n=166)-9.5 \mathrm{mmHg}$ at 12 weeks, $-10.3 \mathrm{mmHg}$ at 48 weeks; Arrhythmia $(n=64)$ $-10.1 \mathrm{mmHg}$ at 12 weeks, $-13.8 \mathrm{mmHg}$ at 48 weeks (all $p<0.01)$; Valvular $(n=34)-7.9 \mathrm{mmHg}$ at 12 weeks, $-9.8 \mathrm{mmHg}$ at 48 weeks; Non-cardiac $(n=47)$ $-6.6 \mathrm{mmHg}$ at 12 weeks, $-4.2 \mathrm{mmHg}$ at 48 weeks $\mathrm{mmHg}$ (both $p<0.05$ ).

$97.5 \%$ of participants completed the initial 12-week programme with $35.1 \%$ opting to stay for the full 48 weeks.

Conclusions: A 12-week lifestyle intervention significantly reduced SBP, and this effect was maintained or further improved at 1-year. The effect was most powerful in cardiac populations.

Disclosure: None

\section{References:}

1. Ettehad D, Emdin CA, Kiran A, Anderson SG, Callender $\mathrm{T}$, Emberson $\mathrm{J}$, et al. Blood pressure lowering for prevention for cardiovascular disease and death: a systemic review and meta-analysis. Lancet. 2015;387(10022):957-67.

0-16 Virtual hypertension clinic: integrating primary and secondary hypertension care in North Central London

Stephen Walsh ${ }^{* 1}$, Reecha Sofat ${ }^{1}$, Jennifer Cross ${ }^{1}$
${ }^{1}$ University College London, London, United Kingdom

Introduction: Hypertensive patients benefit from early control of BP to ameliorate end-organ damage. However, access to specialist care for hypertensive patients can involve long waiting times, travel and expense for patients and CCGs. In 2016, we developed a virtual hypertension clinic in collaboration with Camden CCG to provide remote advice to GPs and triage hypertension patients to community nurse led or hospital clinics.

Methods: Two consultant nephrologists (SBW, JMC) review referrals made to the virtual hypertension clinic on the EMISWeb platform every week. EMISWeb is an electronic patient record system used to record all the clinical observations, measurements and investigation results for all patients seen in Camden primary care. At the virtual clinic, the referral, and the patient's records can be viewed with clinic $\mathrm{BP}$ and ABPM measurements, medications (and number of collected prescriptions), biochemistry and other special investigations and medical history. A treatment plan is written at the time of review for the GP. This may be for: 2 Simple advice with a plan for pharmacotherapy with BP goals.

- Direct triage to a community hypertension nurse specialist clinic for longer consultations than possible for GPs. This includes motivational interviewing, stress workshops, lifestyle and dietary advice.

- Direct triage to hospital clinic for patients who require investigation for secondary hypertension or adherence testing.

Results: Since 2016, 846 patients (median age 43 years, $53 \%$ male) were reviewed.

Referral indications: Age of onset $<40 \mathrm{yrs} 57 \%$, Resistant hypertension $28 \%$, Unusual hypertension $11 \%$, Other $3 \%$. Outcomes: $35 \%$ discharged with treatment plan, $30 \%$ triaged to community nurse-led clinic, $24 \%$ triaged to hospital clinic, $11 \%$ other outcome. The virtual hypertension clinic provided a minimum cost saving of $£ 112.23$ per patient attendance compared with traditional outpatient care.

Conclusions: Implementation of a virtual hypertension clinic offers a cost-effective, rapid referral mechanism for specialist advice, patient assessment and triage.

Disclosure: None

0-17 Inter-arm blood pressure difference: insights into aetiology from the INTERPRESS-IPD collaboration

Christopher Clark $^{*}$, Fiona Warren ${ }^{I}$, Kate Boddy ${ }^{I}$, Sinead McDonagh $^{1}$, Sarah Moore ${ }^{1}$, Lyne Cloutier ${ }^{2}$, Rod Taylor ${ }^{1}$, Angela Shore $^{1}$, Richard McManus ${ }^{3}$, Victor Aboyans ${ }^{4}$ 
${ }^{1}$ University of Exeter, Exeter, United Kingdom, ${ }^{2}$ Université du Québec à Trois-Rivières, Trois-Rivières, QC, Canada, ${ }^{3}$ University of Oxford, Oxford, United Kingdom, ${ }^{4}$ Dupuytren University Hospital, Limoges, France

Introduction: Differences in systolic blood pressure (BP) between arms are associated with excess mortality and cardiovascular events. A systolic inter-arm difference (IAD) has been attributed to subclavian stenosis and arterial stiffening, but the patho-physiological basis of IAD has not been clearly established. We investigated cross-sectional associations of systolic IAD within the INTERPRESS individual participant data (IPD) Collaboration to gain further insight.

Methods: We explored univariable and multivariable models, examining cross-sectional associations of IAD with markers of cardiovascular risk, using hierarchical IPD linear regression, with random effects for study. IAD was calculated from a single sequential pair of BP readings. Model comparison using Aikeke's information criterion (AIC) and likelihood ratios (LR) was undertaken.

Results: The INTERPRESS-IPD Collaboration includes over 57,000 IPD records from 24 studies [mean (SD), age 60.3 (12.5); 47\% female; mean BP 139/81 (22/12)]. Baseline prevalences of systolic IADs $\geq 10 \mathrm{mmHg}$ and $\geq 15 \mathrm{mmHg}$ were $28.2 \%$ and $11.1 \%$, respectively. Using complete case IPD ( $n=43,488 ; 22$ cohorlts), IAD was larger in females $(p=0.002)$, with smoking $(p=0.04)$, increasing age $(p=0.05)$, body mass index $(p<0.001)$, systolic BP $(p<0.001)$ and hypertension $(p<0.001)$. IAD was smaller for African American $(p=0.04)$ and Hispanic American $(p<0.001)$ participants compared to White ethnicity.

Using all available IPD, goodness of fit improved after taking account of pre-existing cardiovascular disease (LR $p=0.004 ; n=41,664$ ), addition of pulse pressure (LR $p<0.001 ; n=33,844$ ) or adjustment for renal disease (LR $p<0.001 ; n=15,541$ ). AIC was non-discriminatory between models.

Conclusions: This analysis confirms independent crosssectional associations of systolic IAD with recognised cardiovascular risk markers. Findings are consistent with hypotheses that IAD reflects pathological change in the circulation. IAD risk factors are those associated with both arterial stiffening and occlusive arterial disease; both processes may contribute to IAD. These findings support further hypothesis generation and justify studies to further elucidate the aetiology of IAD.

Disclosure: None

\section{O-18 Omitted}

P-01 Referrals to acute medicine for 'Hypertension': are these true hypertensive crises, and are we managing them appropriately?

Tehreem Butt ${ }^{1}$, Kanatheepan Shanmuganathan ${ }^{I}$, Daniel Hodges $^{* I}$, Natasha Khan ${ }^{I}$, Mahima Shetty ${ }^{I}$

${ }^{1}$ Broomfield Hospital, Chelmsford, United Kingdom

Introduction: Hypertension has been reaffirmed as the single biggest risk factor contributing to death rates worldwide. General practitioners play a crucial role in management; however, this challenge may also present to the acute physician in the form of a 'hypertensive crisis'. Currently, there is little data examining such patients presenting to acute medical services. We aimed to determine whether patients referred to either the ambulatory care unit (ACU) or acute medical Unit (AMU) with 'hypertension', are true hypertensive crises, to characterise this group of patients demographically, and to determine whether their management is in accordance to existing guidance.

Methods: A retrospective analysis of case notes and electronic records of patients identified as having 'Hypertension' as their primary diagnosis over a 12 month period (Jan 2017-Dec 2017) was undertaken. Data collected included source of referral, blood pressure (BP) at presentation, investigations undertaken, treatment, and followup arrangements.

Results: Fifty-eight patients were included in the audit. Mean age was 62 years with age range of 29-86 years. $51.7 \%$ were referred from primary care, and $34.4 \%$ from the emergency department (ED). Mean systolic BP at presentation was $217.3 \mathrm{mmHg}$ and mean diastolic BP $116 \mathrm{mmHg}$. Most patients met criteria for a 'Hypertensive crisis' (94.7\%). Investigations for end-organ damage were undertaken inconsistently (e.g. fundoscopy $36.2 \%$ vs. renal function tests $82.7 \%$ ). Although only $69 \%$ were treated in accordance to NICE guidelines ${ }^{2}$, reasons such as drug intolerance were not always recorded. Most (95\%) were followed up (e.g. by GP or specialist hypertension clinic).

Conclusions: Referrals to ACU and AMU for hypertension were appropriate. Although investigations to assess end-organ damage were inconsistently performed, most were referred for follow-up where these may have been subsequently undertaken. Further work is needed to assess outcomes in such patients, including efficacy of treatment in achieving BP control.

Disclosure: None 
P-02 Ever increasing referrals: virtually vanish

\section{Andrea Llano ${ }^{* 1}$, Karen Campbell ${ }^{I}$, Marie Freel ${ }^{I}$, Scott Muir $^{1}$}

${ }^{1}$ Queen Elizabeth University Hospital, Glasgow, United Kingdom

Introduction: Changes in hypertension guidelines have resulted in an increase in secondary care referrals and consequent delays in first clinic appointments. A nurse led service was established to undertake targeted investigations prior to clinic attendance in order to streamline the patient journey. We proposed a virtual clinic process whereby these results were reviewed by senior clinicians and so reduce unnecessary out-patient clinic appointments.

Methods: We reviewed referrals to the Glasgow Blood Pressure Clinic (GBPC) between December 2016 and March 2017 when all patients were appointed directly to clinic. We compared these with referrals between September and December 2018 which were reviewed in the virtual clinic. We collected data including reason for referral, time to first appointment and new appointments saved as a result of this process.

Results: There were 142 referrals to the GBPC between December 16-March 2017. 64.8\% (92) of referrals were for specialist review, $14.8 \%$ (21) were for ambulatory blood pressure monitoring (ABPM) and $19.7 \%$ (28) were for resistant hypertension or patients with multiple intolerances. 1 patient was discharged prior to clinic review following ABPM review. The remaining 141 patients were all given clinic appointments and $22.5 \%$ of referrals were discharged from first clinic visit.

There were 94 referrals between September and December 2018. 62.8\% (59) of referrals were for specialist review, $28.7 \%$ (27) were for ABPM and $7.5 \%$ (7) were for resistant hypertension or patients with multiple intolerances. $36 \%$ (34) of referrals were discharged from the service prior to clinic attendance. GPs were provided with ABPM results, reasons for discharge from the service and advice where appropriate.

Conclusions: Review of ABPM results prior to clinic attendance has led to a reduction in the number of unnecessary new patient clinic appointments and more timely management of hypertension referrals from primary care.

Disclosure: None

P-03 Missed opportunities: An audit of blood pressure measurements in the cardiology outpatient department
${ }^{1}$ Wythenshawe Hospital, Manchester, United Kingdom,

${ }^{2}$ Stepping Hill Hospital, Stockport, United Kingdom

Introduction: Hypertension causes a significant public health burden in the United Kingdom and worldwide, thus it is vital to ensure that raised blood pressure is acted on appropriately. The National Institute for Health and Care Excellence (NICE) have produced a clear set of guidelines outlining how hypertension should be assessed and managed and at what blood pressure levels intervention should be started at.

The aim of this audit was to assess whether blood pressure (BP) was being measured in cardiology outpatient clinics at a District General Hospital and when raised blood pressures were identified, were they acted on appropriately.

Methods: 200 consecutive cardiology outpatient clinic records of patients seen between 1st and 12th September 2017 were accessed from an electronic database to assess whether BP had been measured and what action had been taken if hypertension was identified.

Results: All patients had BP measured by a nurse but only those seen by a hypertension specialist had further measurements. 33\% (66) had raised BP ( $\geq 140 / 90$, or $\geq 150$ / 90 if age $\geq 80$ years). Of these $61 \%$ (40) had further management or were referred to their GP for this.

Conclusions: There was a high prevalence of BP elevation in these cardiology outpatients who are more likely to benefit from assessment and possible intervention. The opportunity to manage raised $\mathrm{BP}$, although measured universally was neglected in a significant number of patients. It is appropriate to ensure that cardiologists recognise the importance of acting upon all raised BP measurements to minimise the avoidable consequences of hypertension in their especially vulnerable patients.

Disclosure: none

Database clarification:The database used was the Evolve system to look at clinic letters. With regards to the conclusions all the conclusions were made from the results section of the abstract with exception of saying their was a high prevalence, however this can be concluded by the prevalence of $33 \%$ in the outpatient population compared to the UK average of estimated $18 \%$ according to the Health survey England in 2017.

\section{P-04 Prevalence of masked hypertension in treated and untreated clinically normotensive patients}

\section{Kamran Basharat ${ }^{* 1}$, Tarek Antonios ${ }^{1}$}

${ }^{1}$ St George's, University of London, London, United Kingdom

\section{Guy Kendall ${ }^{* 1}$, Philip Lewis ${ }^{2}$}


Introduction: Masked hypertension (MH) and masked uncontrolled hypertension (MUCH) are both associated with increased cardiovascular disease risk. However, few data is available on the prevalence MH and MUCH in the UK population. Likewise, there is limited data on the characteristics of the MH subtypes of masked daytime hypertension (MDH) and masked nocturnal hypertension (MNH).

Methods: In this retrospective study, we analysed the data of 177 individuals with non-elevated clinic BP $(\mathrm{CBP}<$ $140 / 90 \mathrm{mmHg}$ ) who attended our clinic for 24-h ambulatory $\mathrm{BP}$ monitoring (ABPM). 105 of these individuals were taking antihypertensive medication whilst 72 were not. A Spacelabs 90217 BP Monitor was used to asses ambulatory $\mathrm{BP}$ (ABP). Individuals with a non-elevated CBP, nonelevated daytime $\mathrm{ABP}(\mathrm{BP}<135 / 85)$ and non-elevated nocturnal $\mathrm{BP}(\mathrm{BP}<120 / 70)$ were classified as having sustained normotension ( $\mathrm{SN})$, or controlled $\mathrm{BP}$ if on antihypertensive medication. Individuals with a non-elevated CBP but with an elevated daytime ABP (SBP $\geq 135$ and/or $\mathrm{DBP}) 285$ ) were diagnosed with $\mathrm{MDH}$, or masked uncontrolled daytime hypertension (MUCDH) if taking antihypertensive medication. individuals with a non-elevated $\mathrm{CBP}$, a non-elevated daytime $\mathrm{ABP}$, but an elevated nocturnal $\mathrm{ABP}$ ( $\mathrm{SBP} \geq 120$ and/or $\mathrm{DBP} \geq 70$ ) were diagnosed as having $\mathrm{MNH}$, or $\mathrm{MUCNH}$ if taking antihypertensive medication.

Results: Of those untreated, 40 patients (55.6\%) were diagnosed with MH (age 42.3 years, $57.5 \%$ men). Of those taking medication, 74 patients (70.5\%) were diagnosed with MUCH (age 53.4 years, $51.4 \%$ men). Smoking history was significantly higher in $\mathrm{MDH}$ and $\mathrm{MNH}$ groups. The proportion of individuals with high-normal CBP $(\geq 130 / 85$ but $<140 / 90)$ was also significantly higher in $\mathrm{MDH}(p<0.001)$, MNH $(p<0.048)$ and MUCDH groups $(p<0.001)$.

Conclusions: The prevalence of MH and MUCH is high in individuals attending a specialist hypertension centre. Our findings suggest the need for the acknowledgement of these entities by UK guidelines, and suggest $24 \mathrm{~h}-\mathrm{ABPM}$ (rather than day-time) screening in individuals with highnormal $\mathrm{BP}$ and in smokers.

Disclosure: None

P-05 Under-recognised and undertreated hypertension (HTN) in patients with obstructive sleep apnoea (OSA)

\author{
Awolkhier Mohammedseid Nurhussien ${ }^{* 1}$, Francesco \\ P. Cappuccio ${ }^{1}$, Asad Ali ${ }^{1}$ \\ ${ }^{1}$ University Hospitals Coventry \& Warwickshire NHS \\ Trust, Coventry, United Kingdom
}

Introduction: Obstructive Sleep Apnoea is a common and treatable condition, but up to $80 \%$ of people with OSA have not yet been diagnosed [1]. The prevalence of HTN in OSA patients ranges from 35 to $80 \%$ and appears to be influenced by OSA severity [2]. Severe untreated OSA has been linked to fatal and nonfatal cardiovascular events, and all-cause mortality [3]. Hypertension secondary to OSA is underrecognised and undertreated.

Methods: We undertook a clinical audit as part of quality improvement project with retrospective review of electronic records in our Trust. NICE and ESH hypertension guidelines used as standard.

Results: 423 patients referred for sleep study were studied and $75 \%$ confirmed to have OSA of which $53 \%$ with moderate to severe OSA.105 patients with diagnosis of severe OSA were analysed further and $62(59 \%)$ patients had diagnosis of hypertension. 43 patients with severe OSA had no screening for HTN. 11 patients with diagnosis of hypertension and severe OSA were not on any medical treatment. 12 patients were not on optimal target blood pressure control. $60 \%$ of patients who were taking 3 or more antihypertensive medications were not on target and not under specialist care.

Conclusions: This clinical audit highlights that patients with OSA are under-recognised and undertreated for their hypertension. Currently we are undertaking quality improvement project on how to improve awareness, develop screening and referral pathways for these patients. Further study is required for guidance on management of OSA with HTN.

Disclosure: None

\section{References:}

1. www.blf.org.uk. 2019.OSA https://www.blf.org.uk/ sites/default/files/BLF_OSA-Top-Tips-for-GPs_ DOWNLOAD.pdf

2. Calhoun DA. Obstructive sleep apnea and hypertension. Curr Hypertens Rep. 2010;12:189-95.

3. Marshall NS, Wong KK, Liu PY, et al. Sleep apnea as an independent risk factor for all-cause mortality: the Busselton Health Study. Sleep. 2008;31:1079-85.

P-06 Hypertension in HIV and comparison to other high risk groups

Gavin Manmathan $^{* 1,2}$, Nkemamaka Okonkwo ${ }^{1,2}$, Robin Ramphul $^{3}$, Abdul Zubairu ${ }^{4}$,Ming Lee ${ }^{5}$, Moses Shongwe $\boldsymbol{6}^{6}$, Faith Odong $^{7}$, Thembeka Moyo ${ }^{7}$, Sophia Rein ${ }^{2}$, Tristan Barber $^{1}$, Sabine Kilnoch ${ }^{1}$, Fiona Burns ${ }^{1,2}$, Margaret Johnson $^{1,2}$, Roby Rakhit ${ }^{1,2}$

${ }^{1}$ Royal Free Hospital, London, United Kingdom, ${ }^{2}$ University College, London, United Kingdom, ${ }^{3}$ St Helier's 
Hospital, London, United Kingdom, ${ }^{4}$ Norwood GP Surgery, Southport, United Kingdom, ${ }^{5}$ Guys and St. Thomas' Hospital, London, United Kingdom, ${ }^{6}$ St. Bartholomew's Hospital, London, United Kingdom, ${ }^{7}$ Lewisham Hospital, London, United Kingdom

Introduction: People living with HIV (PLWH) have 1.5-2.2fold increased relative risk of cardiovascular events. American College of Cardiology (ACC) updated guidelines in 2018 to recommend a lowered blood pressure $(\mathrm{BP})$ treatment threshold of 130/80 from 140/90 $\mathrm{mmHg}$ [1]. UK guidelines are due to be addressed in 2019. We aim to compare blood pressure differences in PLWH with patient cohorts accessing other healthcare settings (renal clinic and general practice (GP).

Methods: Prospective data collection from 4 HIV units, GP surgery, and a renal unit (CKD stage 3-5 excluding dialysis patients) over one week. Age, sex, BP, BP medication, previous heart attack (MI) or stroke (CVA), years on antiretroviral therapy (ART) were collected.

Results: 431 people included, 271 PLWH (mean 47 years, mean ART duration 11 years), 41 from GP (59 years), 119 from renal (56 years). 265 (61\%) male, 235 (55\%) white, 137 (32\%) black African/Caribbean, Asian 35 (8\%).

Of 100 people on BP medication $56 \%$ had uncontrolled BP (>140/90). Mean systolic BP in PLWH 132; comparable to GP $(134 ; p=0.78)$ but lower than renal $(139 ; p<0.01)$. $13 \%$ of GP patients were on BP medications; $15 \%$ of PLWH, $28 \%$ in renal. In PLWH $45 \%$ were uncontrolled. 62 (29\%) PLWH not on medication had high BP (>140/90) which would increase to $133(62 \%)$ using ACC guidelines. Prevalence of MI/CVA was higher in PLWH (5.5\%) compared to GP (4.9\%).

Conclusions: PLWH represent a vulnerable group of younger patients with an increasing incidence of cardiovascular risk. They have high prevalence of undiagnosed hypertension and, of those on BP treatment, a significant number were uncontrolled. We advocate using the ACC guidelines to diagnose and treat PLWH to reduce cardiovascular morbidity and mortality.

Disclosure: None

References:

1. Dominiczak AF, Kuo D. Hypertension. 2018;71(1):3-4.

\section{P-07 The management of post-partum pre-eclampsia: a case report series}

\section{Olivia Meakin', Moulinath Banerjee ${ }^{* 1}$, Neeraja Singh ${ }^{1}$ \\ ${ }^{1}$ Royal Bolton Hospital, Bolton, United Kingdom}

Introduction: Ante- and intra-partum preeclampsia is a common condition that is managed in all obstetric departments nationwide. Postpartum pre-eclampsia is significantly less common, therefore its management is less clearly documented and less evidence-based. The treatment is either using the drugs used during pregnancy, or the ones suggested by NICE-BHS guidelines.

Methods: Two female patients, with no history of preeclampsia, were noted to be suffering from refractory hypertension post-partum. Their hypertension did not resolve with conventional obstetric medical management, including labetalol, hydralazine and magnesium sulphate, therefore endocrinological advice was sought. A case report series was formulated to highlight the successful outcomes, good clinical practice, and the need for improved knowledge and guidelines surrounding post-partum preeclampsia.

Results: We found that in women who are not breastfeeding, their hypertension, resistant to other agents, responded better to cardio-selective beta-blockers, such as bisoprolol. Both patients had their hypertension successfully managed with cardio-selective beta-blockers +/calcium channel blockers and alpha-antagonists, and have been well since. Women who are breastfeeding should be treated with the drugs, which are known to be safe for the infant. Women who are not breastfeeding, may be treated with cardio-selective beta-blocker based therapy, which will be helpful due to their once daily dosing to fit in to the busy life of a parent.

Conclusions: Cardio-selective beta-blocker based therapy may benefit young women with post-partum pre-eclampsia. Very little is understood surrounding the pathophysiology of post-partum pre-eclampsia and its management, therefore making it a very difficult disease to manage when seen in the clinical setting. Further research in the field is required to better understand the nature of the disease, and to improve the quality of care we can offer our patients.

Disclosure: None

\section{P-08 Addressing hypertension in pregnancy in Western Ghana: the akuba foundation}

\section{Adam Greenstein ${ }^{* 1}$, Garry Sanderson', Jecko Thachil', Jenny Oliver ${ }^{3}$, Paul Robinson ${ }^{4}$}

${ }^{1}$ University Of Manchester, Manchester, United Kingdom,

${ }^{2}$ Manchester University Foundation Hospitals NHS Trust, Manchester, United Kingdom, ${ }^{3}$ The Akuba Foundation, Manchester, United Kingdom, ${ }^{4}$ Queen Mary University of London, London, United Kingdom

Introduction: In 2017, a patient from the Hypertension clinic at Manchester Royal Infirmary requested assistance with the delivery of care for pregnant women with BP- 
related complications in Western Ghana. A fact-finding team was assembled in 2018, comprising hypertension physicians (AG, PR), a haematologist (JT), business strategy consultant (GS) and a charitable sector startup executive (JO). We gratefully acknowledge funding contributions from BIHS, Manchester University Social Responsibility programme and the generosity of our local hosts.

Methods: We spent 5 days in the Western Region of Ghana, visiting rural villages to evaluate local healthcare provision. We met with health visitors and midwives and learned of their experiences and outlooks for the treatment of (pre)eclampsia. We visited three hospitals, met with management teams and clinicians who deliver maternity care, and we attended a novel 'Pregnancy School'.

Results: We will present an overview of the current provision for hypertension care in the setting of pregnancy within Western Ghana. Qualitative evaluation with midwives revealed enthusiasm for health promotion, and good levels of education and awareness, but concerns regarding parallel pathways for pregnancy management by untrained 'Traditional Birthing Attendants (TBA), the availability of equipment and medication, and the capacity for patients to attend care due to lack of affordable transport. We describe innovative recent schemes to facilitate collaboration and cross-talk between the TBA approach and modern obstetrics and midwifery.

Conclusions: The sub-optimal care of hypertension in pregnancy is a complex interaction of social beliefs (use of herbal remedies, TBAs, gender roles in society), poverty (despite care being free at the point of need, in theory) and the adequate provision of western healthcare (both manpower and facilities). Tackling one without the others is unlikely to produce sustained benefit, however, there is significant opportunity to improve outcomes with a multifactorial approach.

Disclosure: None

\section{P-09 Medium term haemodynamic and blood pressure effects of living kidney donation}

\author{
Anna Price ${ }^{* 1,2}$, Jonathan Law ${ }^{1,2}$, Manvir Hayer ${ }^{2}$, Luke \\ Pickup $^{2}$, Ashwin Radhakrishnan ${ }^{2}$, William Moody ${ }^{2}$, \\ Nicola Edwards ${ }^{2}$, Charles Ferro ${ }^{1,2}$, Jonathan Townend ${ }^{1,2}$
}

${ }^{1}$ Queen Elizabeth Hospital Birmingham, Birmingham, United Kingdom, ${ }^{2}$ Birmingham Cardio-Renal Research Group, Birmingham, United Kingdom

Introduction: Current guidance encourages clinicians to counsel living kidney donors that blood pressure may rise following nephrectomy $[1,2]$. A meta-analysis estimated an increase of $6 \mathrm{mmHg}$ in systolic and $4 \mathrm{mmHg}$ in diastolic blood pressure five years after donation [3]. There are however, few long term prospective controlled studies.

Methods: Living kidney donors and healthy controls recruited to the CRIB-DONOR study between 2011 and 2013 were followed up at 5 years [4]. Participants underwent 24-hr ambulatory blood pressure, augmentation index and pulse wave velocity using the Mobli-O-Graph ${ }^{\circledR}$.

Results: Ninety four patients consented $(n=92$ valid day recordings, $n=74$ valid night recordings). ${ }^{5}$ There was no significant difference in 24-h ambulatory systolic readings in donors compared to controls $119.97 \pm 9.35$ vs $119.68 \pm 9.34 \mathrm{mmHg}, \quad p=0.879$ or diastolic readings $75.79 \pm 8.31$ vs $75.54 \pm 7.99 \mathrm{mmHg}, p=0.885$.

Measures of arterial stiffness were also comparable between the two groups. Pulse wave velocity was not significantly different in donors compared to controls $7.63 \pm$ 1.52 vs $7.27 \pm 1.52 \mathrm{~m} / \mathrm{s}, p=0.242$. There was also no difference in augmentation index (corrected to 75 beats per minute) between donors and controls $23.14 \pm 8.06$ vs $21.82 \pm 8.22 \%, p=0.441$.

Conclusions: In this small cohort kidney donors and clinicians can be reassured that there is no significant rise in blood pressure or change in arterial function.

Disclosure: No conflicts of interest.

\section{References:}

1. Lentine KL, Kasiske BL, Levey AS, Adams PL, Alberú J, Bakr MA, et al. Kdigo clinical practice guideline on the evaluation and care of living kidney donors. Transplantation. 2017;101:S7-S105.

2. Abramowicz D, Cochat P, Claas FHJ, Heemann U, Pascual J, Dudley C, et al. European renal best practice guideline on kidney donor and recipient evaluation and perioperative care. Nephrol Dial Transplant. 2015;30:1790-97.

3. Boudville N, Ramesh Prasad GV, Knoll G, Muirhead $\mathrm{N}$, Thiessen-Philbrook H, et al. Meta-analysis: risk for hypertension in living kidney donors. An Int Med. 2006;145:185-96.

4. Moody WE, Ferro CJ, Edwards NC, Chue CD, Lin ELS, Taylor RJ, et al. Cardiovascular effects of unilateral nephrectomy in living kidney donors. Hypertension. 2016;67:368-77.

5. Parati G, Stergiou G, O’Brien E, Asmar R, Beilin L, Bilo G, et al. European society of hypertension practice guidelines for ambulatory blood pressure monitoring. J Hypertens. 2014;32:1359-66. 
P-10 Nocturnal dipping and blood pressure variability in living kidney donors five years after nephrectomy

Anna Price ${ }^{* 1,2}$, Luke Pickup ${ }^{2}$, Ashwin Radhakrishan ${ }^{2}$, Jonathan Law ${ }^{1,2}$, Manvir Hayer ${ }^{2}$, William Moody ${ }^{2}$, Nicola Edward $^{2}$, Charles Ferro ${ }^{1,2}$, Jonathan Townend ${ }^{1,2}$

${ }^{1}$ Queen Elizabeth Hospital Birmingham, Birmingham, United Kingdom, ${ }^{2}$ Birmingham Cardio-Renal Research Group, Birmingham, United Kingdom

Introduction: Recent studies indicate that living kidney donors may have an increased risk of cardiovascular mortality and demonstrate subtle changes in cardiovascular structure including elevated left ventricular mass after nephrectomy [1-3]. Blood pressure, however, at least in the short term, does not appear to change [1,4]. In chronic kidney disease nocturnal blood pressure is a superior predictor of cardiovascular mortality than day or 24-h blood pressure results [5]. We sought to investigate whether there are changes in nocturnal dipping and blood pressure variability in donors.

Methods: Healthy controls and living kidney donors recruited to the CRIB-DONOR study were followed up at 5 years. ${ }^{1}$ All participants underwent $24-\mathrm{h}$ ambulatory blood pressure recordings using the Mobli-O-Graph ${ }^{\circledast}$. Nocturnal profiles were defined according to the percentage decline in blood pressure $(<0 \%$ inverted; $<10 \%$ Non-dipper; $<20 \%$ Normal; $>=20 \%$ extreme).

Blood pressure variability was calculated using previously published calculations for average real variability, weighted blood pressure and day-night difference [6-8]. Recordings with a gap of greater than two hours of consecutive recordings were excluded from variability analysis.

Results: A total of 94 patients consented with 74 adequate night recordings for analysis [9]. There was no significant difference in nocturnal dipping in systolic blood pressure between donors and controls $11.20 \pm 5.53$ vs $9.27 \pm 6.20 \%, p=0.381$. There was also no significant difference in nocturnal dipping in diastolic blood pressure between donors and controls $14.62 \pm 5.87$ vs $13.40 \pm$ $5.81 \%, p=0.162$.

Only 6/41 (14\%) of donors had non-dipping or inverted profiles, the majority were normal. There was no difference in nocturnal profiles, average real variability, weighted blood pressure variability or day night difference between donors and controls.

Conclusions: Based on these results with a modest sample size donors can be reassured that there is little evidence of abnormal nocturnal profiles or blood pressure variability at 5 years.

Disclosure: No conflicts of interest.

\section{References:}

1. Moody WE, Ferro CJ, Edwards NC, Chue CD, Lin ELS, Taylor RJ, et al. Cardiovascular effects of unilateral nephrectomy in living kidney donors. Hypertension. 2016;67:368-77.

2. Altmann U, Böger CA, Farkas S, Mack M, Luchner A, Hamer OW, et al. Effects of reduced kidney function because of living kidney donation on left ventricular mass. Hypertension. 2017;69:297-303.

3. Mjoen G, Hallan S, Hartmann A, Foss A, Midtvedt K, Oyen $\mathrm{O}$, et al. Long-term risks for kidney donors. Kidney Int. 2014;86:162-67.

4. Kasiske BL, Anderson-Haag T, Israni AK, Kalil RS, Kimmel PL, Kraus ES, et al. A prospective controlled study of living kidney donors: three-year follow-up. Am J Kidney Dis. 2015;66:114-24.

5. Minutolo R, Agarwal R, Borrelli S, Chiodini P, Bellizzi V, Nappi F, et al. Prognostic role of ambulatory blood pressure measurement in patients with nondialysis chronic kidney diseaseprognostic role of ambulatory bp measurement. Arch Int Med. 2011;171:1090-98.

6. Bilo G, Giglio A, Styczkiewicz K, Caldara G, Maronati A, Kawecka-Jaszcz K, et al. A new method for assessing 24-h blood pressure variability after excluding the contribution of nocturnal blood pressure fall. J Hypertens. 2007;25:2058-66.

7. Mule G, Calcaterra I, Costanzo M, Morreale M, D'Ignoto F, Castiglia A, et al. Average real variability of 24-h systolic blood pressure is associated with microalbuminuria in patients with primary hypertension. J Hum Hypertens. 2016;30:164-70

8. Musameh MD, et al. Determinants of day-night difference in blood pressure, a comparison with determinants of daytime and night-time blood pressure. J Human Hypertens. 2017;31:43-8.

9. Musameh MD, Nelson CP, Gracey J, Tobin M, Tomaszewski M, Samani NJ. European society of hypertension practice guidelines for ambulatory blood pressure monitoring. J Hypertens. 2014;32:1359-66.

P-11 Home blood pressure measurement in the treatment in morning vs. evening (TIME) study

\section{Amy Rogers ${ }^{* 1}$, Xiaodan $\mathrm{Li}^{2}$, David Rorie ${ }^{1}$, Isla Mackenzie $^{I}$, Thomas MacDonald ${ }^{I}$}

${ }^{1}$ University of Dundee, Dundee, United Kingdom, ${ }^{2}$ Sichuan University, Chengdu, China

Introduction: The Treatment in Morning vs Evening (TIME) study is a large online prospective study comparing cardiovascular outcomes in 21,104 hypertensive people 
randomised to taking their usual anti-hypertensive medications in either the morning or the evening [1]. The study began in 2011 and continues in follow-up. Participants who reported owning their own home blood pressure monitor (HBPM) were invited to submit HBPM measurements at intervals throughout the study. We describe the home BP data collected in the TIME study.

Methods: TIME study participants who agreed to submit HBPM measurements were asked to submit sets of measurements (comprising 3 measurements, morning and evening, for 4-7 days) at baseline, 1 month, and 3 monthly thereafter. Data were entered directly into the study database by participants via a secure webpage. Means were compared using dependent $\mathrm{t}$-testing ( $\mathrm{R}$ version 3.5.2).

Results: 11,166 TIME participants were willing to submit HBPM measurements and 11,126 submitted any HBPM data. Participants have so far submitted a median of 4 sets of HBPM measurements (range 0-26). 6484 participants continue to submit regular sets of measurements. 6946 participants $(62.4 \%)$ submitted sufficient data to calculate mean baseline morning and evening blood pressures. Mean overall BP at baseline was $133 / 77( \pm 12 / 8) \mathrm{mmHg}(132 / 78$ morning, $133 / 77$ evening). $63.1 \%$ of participants reported baseline systolic BP of $<135 \mathrm{mmHg}$. Mean overall BP at 2 years was $130 / 76( \pm 11 / 8) \mathrm{mmHg}$ with $70 \%<135 \mathrm{mmHg}$ systolic. On average, systolic BP at 2 years was significantly lower than at baseline $(t=-8.57, p<0.05)$.

Conclusions: The TIME study collected HBPM data using participants' own monitors. Participants were willing and able to submit sufficient measurements to allow calculation of mean morning and evening blood pressures. These data will allow further insight into the outcomes of the TIME study.

Disclosure: The TIME study is funded by the British Heart Foundation and supported by the BIHS.

\section{References:}

1. Rorie DA, Rogers A, Mackenzie IS, Ford I, Webb DJ, Williams B, et al. Methods of a large prospective, randomised, open-label, blinded end-point study comparing morning versus evening dosing in hypertensive patients: the Treatment In Morning versus Evening (TIME) study. BMJ Open. 2016;6. http:// bmjopen.bmj.com/content/6/2/e010313.abstract

\section{P-12 Omitted \\ P-13 Omitted}

P-14 Effects of slow breathing and mental stress on the cardiac component of the baroreflex in young adults with hypertensive parents $(\mathrm{FH}+)$ compared to those with normotensive parents $(\mathbf{F H}-)$

\section{Fahad Al Enazi ${ }^{* 1}$, Andrew Coney ${ }^{I}$, Janice Marshall ${ }^{1}$}

${ }^{1}$ University of Birmingham, Birmingham, United Kingdom

Introduction: White Europeans (WEs) and South Asian (SAs) with hypertensive parents $(\mathrm{FH}+$ ) have greater risk of hypertension than those with normotensive parents $(\mathrm{FH}-)$. Whether autonomic control of arterial blood pressure (ABP) is impaired in young normotensive $\mathrm{FH}+$ is unclear.

Methods: In $8 \mathrm{FH}-$ and $8 \mathrm{FH}+(4 \mathrm{WE} / 4 \mathrm{SA} ; 4 \mathrm{men} / 4$ women in each group; 18-25 years), we recorded ABP, ECG, respiration, baroreflex sensitivity (BRS) by sequence analysis and squat-stand test, heart rate variability (HRV) by spectral analysis, at rest, during slow breathing ( 6 breaths/ min), and mental stress (Stroop test).

Results: $\mathrm{FH}-$ and $\mathrm{FH}+$ had similar mean $\mathrm{ABP}$ (mABP: $78.5 \pm 3.2$ vs $83.6 \pm 3.4 \mathrm{mmHg})$ and respiration $(15.7 \pm 1.3$ vs $16.2 \pm 0.7$ breaths $/ \mathrm{min}$ ) at rest. Slow breathing increased BRS during up-sequences in $\mathrm{FH}+(1.2 \pm 0.06$ to $1.3 \pm$ $0.06 * \mathrm{~ms} / \mathrm{mmHg}, *: P<0.05)$, but not $\mathrm{FH}-(1.21 \pm 0.08$ to $1.35 \pm 0.09 \mathrm{~ms} / \mathrm{mmHg}$ ), and increased the low $/$ high power ratio $(\mathrm{LF} / \mathrm{HF})$ of $\mathrm{HRV}$ in $\mathrm{FH}+$ by increasing $\mathrm{LF}(28.1 \pm 4.9$ to $72.1 \pm 4.4 * \%)$ and decreasing HF $(37.7 \pm 5.1$ to $18.0 \pm$ $2.7 * \%$ ), but by increasing LF only in FH- (LF: $30.2 \pm 2.9$ to $62.0 \pm 5.8 * \%$; HF: $29.9 \pm 6.1$ to $24.6 \pm 5.7 \%$ ). During mental stress, BRS during up-sequences was decreased in both $\mathrm{FH}+$ and $\mathrm{FH}-$, but, BRS tachycardia during squatstand was decreased in $\mathrm{FH}-$ only ( $\mathrm{FH}-0.64 \pm 0.05$ vs $0.26 \pm 0.11^{*} ; \mathrm{FH}+0.48 \pm 0.06$ vs $0.46 \pm 0.09 \mathrm{~ms} / \mathrm{mmHg}$ ).

Conclusions: Slow breathing may benefit young normotensive $\mathrm{FH}+$, increasing the vagal component of BRS and of HRV by increasing vagal coupling with respiratory frequency. However, mental stress may be more detrimental to $\mathrm{FH}+$ : the vagal component of BRS is inhibited as in $\mathrm{FH}-$, but the sympathetic component causes further tachycardia in FH+ only.

Disclosure: None

\section{References:}

1. Wang NY, Young JH, Meoni LA, Ford DE, Erlinger TP. Arch Intern Med. 2008;168:643-8.

2. Ranasinghe P, Cooray DN, Jayawardena R, Katulanda P. BMC Public Health. 2015;15:576.

P-15 Baroreflex regulation of arterial blood pressure is disturbed in young South Asians relative to White Europeans, particularly during mental stress

\section{Mobarak Alqahtani ${ }^{*}$, Andrew Coney ${ }^{I}$, Janice Marshall ${ }^{I}$ \\ ${ }^{1}$ University of Birmingham, Birmingham, United Kingdom}


Introduction: South Asians (SAs) have greater risk of cardiovascular disease than White Europeans (WEs); this is generally associated with endothelial dysfunction [1]. In view of our recent evidence that young SAs show greater sympathetic activation than WEs during mental stress [2], we investigated autonomic regulation of arterial blood pressure (ABP) in both ethnicities.

Methods: In 8 WEs and 8 SAs (4/4 men/women in each group; 19-24 years), ABP, heart rate (HR), ECG and respiration were recorded at rest, mental stress (Stroop test); baroreflex sensitivity (BRS) by sequence method and squatstand test, heart rate variability (HRV) by spectral analysis were calculated.

Results: At rest, WEs and SAs had similar mean ABP (mABP, $82.2 \pm 2.1$ vs. $82.0 \pm 4.2 \mathrm{mmHg})$, HR $(70.1 \pm 3.4$ vs. $64.5 \pm 6.5$ beats $/ \mathrm{min}$ ) and BRS (up-sequence: $1.1 \pm 0.09$, down-sequence: $1.2 \pm 0.05$ vs. $1.2 \pm 0.04$ and $1.3 \pm 0.06 \mathrm{~ms} /$ $\mathrm{mmHg}$ ). However, low/high frequency power ratio (LF/HF) was $1.4 \pm 0.05$ in WEs $(34.0 \pm 6.2 \% / 28.4 \pm 5.1 \%)$, but only $0.7 \pm 0.1$ in SAs $(26.5 \pm 1.7 \% / 41.7 \pm 4.3 \%)$, the HF component tending to be greater in SAs ( $P=0.07$ : SAs vs. WEs). Mental stress evoked similar increases in mABP and HR, but BRS was less depressed in SAs than WE especially during down-sequences (to $0.69 \pm 0.11$ vs. $1.03 \pm 0.05^{*} \mathrm{~ms} / \mathrm{mmHg}$, *: $\mathrm{P}<0.05$, WE vs. SA), while LF was increased in both SAs and WEs (to $42.9 \pm 5.9^{*}$ and $42.8 \pm 4.8 \%$ ), but $\mathrm{HF}$ was decreased in SAs only (to $29.0 \pm 3.4 * \%$ ). Further, immediately following mental stress, BRS during squat-stand was depressed in WEs, but not SAs $(0.6 \pm 0.04$ to $0.3 \pm 0.09 *$ vs. $0.6 \pm 0.09$ to $0.5 \pm 0.11 \mathrm{~ms} / \mathrm{mmHg}$.

Conclusions: Even in young adult SAs, baroreflex regulation of ABP is disturbed particularly during mental stress with SAs showing exaggerated vagally- and sympathetically-mediated tachycardia relative to WEs. This may contribute to their increased risk of hypertension and associated cardiovascular disease

Disclosure: None

References:

1. Gupta M, Singh N, Verma S. Circulation. 2016;113:924-9.

2. Ormshaw NG, Junejo RT, Marshall JM. Eur J Appl Physiol. 2018;118:979-88.

P-16 The effects of sphygmomanometer cuff construction on return-to-flow cuff inflation pressure using a physical simulation model of arm blood flow

\section{Shenela Naqvi', Prasad Potluri', Parthasarathi Mandal, Philip Lewis $^{* 4}$}

${ }^{1}$ NED University of Engineering \& Technology, Karachi, Pakistan, ${ }^{2}$ The University of Manchester, Manchester, UK,
${ }^{3}$ School of Mechanical, Civil and Aerospace Engineering, Manchester, UK, ${ }^{4}$ Stockport NHS Foundation Trust, Stockport, UK

Introduction: Sphygmomanometer cuff construction has significant effects on cuff pressure transmission to the underlying artery using Finite Element Analysis [1]. We therefore created an anthropomorphic perfused model arm to simulate the effects of different cuffs on the pressures at which blood flow may return during deflation.

Methods: A cylindrical vulcanised silicone rubber mandrel (circumference $28 \mathrm{~cm}$, length $30 \mathrm{~cm}$ ) with a metal "humerus" and silastic artery, with similar visco-elastic properties to a human arm was perfused with a $35 \%$ glycerine-in-water solution of similar viscosity to human blood using a peristaltic pump delivering a continuous flow of $160 \mathrm{ml} / \mathrm{min}$ (equivalent to brachial artery flow) at $130 \mathrm{mmHg} .37$ examples of 8 different cuffs of similar size were inflated around the mandrel to $350 \mathrm{mmHg}$ and then deflated at $2-3 \mathrm{mmHg} / \mathrm{s}$ to zero. The cuff pressures at interrupted return-to-flow (RtF), at the point of reappearance of continuous uninterrupted but pulsatile flow (PF) and of continuous non-pulsatile flow (CF) were also assessed (which may approximate to K1, K4 and K5 respectively). The physical characteristics of each cuff: elasticity, flexural rigidity and material thickness were assessed.

Results: There are very significant differences in cuff materials and their resultant physical characteristics. RtF pressures were much higher than perfusion pressure (mean 286, range $275-298 \mathrm{mmHg}$ ) and differed between cuffs of similar size but dissimilar construction. The highest RtF pressure was observed in a rubber-bladdered cuff with a woven sleeve and the lowest in a bladderless woven cuff. $\mathrm{PF}$ and $\mathrm{CF}$ pressures also varied between cuffs but with less variability than $\operatorname{RtF}(13$ and $15 c f .23 \mathrm{mmHg}$ ) and not in the same order.

Conclusions: Differing cuff construction affects the pressure at which flow returns in a perfused model arm. It cannot therefore be assumed that all cuffs of similar size give equivalent blood pressure values.

Disclosure: None

References:

1. Naqvi et al. J Ind Text. 2018;47(7):1478-95. https:// doi.org10.1177/1528083717695838.

P-17 Measurement of systolic blood pressure by parents at home using hand-held Doppler device and aneroid sphygmomanometer: a single centre experience

Joanna Newton ${ }^{1}$, Cheentan Singh ${ }^{1}$, Manish Sinha ${ }^{* 1,2}$ 
${ }^{1}$ Evelina London Childrens Hospital, Kings College London, London, United Kingdom, ${ }^{2}$ Kings College London, United Kingdom

Introduction: There are limited data regarding the clinical use of home BP monitoring in infants and young children and older children unable to tolerate 24-h ambulatory BP monitoring (ABPM). We taught parents to perform home doppler assisted systolic BP monitoring (HDBPM) using aneroid sphygmomanometer [1].

Methods: Retrospective review over a 5-year period of all children referred to our tertiary hypertension service in whom parents/carers performed HDBPM. During a planned hospital appointment, parents were shown how to perform HDBPM with monitoring performed over at least two weeks with three measurements in quick succession performed twice a day.

Results: 116 children underwent HDBPM with median (IQR), $2.25(0.83-4.9)$ years, including $29.5 \%(n=34)$, $30 \%(n=35), 22.5 \%(n=26)$ and $18 \%(n=21)$ in children aged $\leq 1,1-\leq 2,2$ years $-\leq 5$ and $>5$ years old respectively. HDBPM was used as the children were too young $69 \%$ $(n=80)$ or thought unlikely to be able to tolerate ABPM in $31 \%(n=36)$ as a result of underlying learning difficulties or neurological disease. Underlying primary diagnosis was renal in $44.8 \%(n=52)$, and non-renal in others. Children had been referred to our service for confirmation of hypertension, $51.7 \%$ (confirmation group, $n=60 / 116)$ or for monitoring of BP levels $48.3 \%$ (monitoring group, $n=56$ ) for those on anti-hypertensive therapy. In the confirmation group, $90 \%(n=54 / 60)$ were found to be normotensive and only $10 \%(n=6 / 60)$ to have confirmed hypertension. In the monitoring group, 94.6\% ( $n=53 / 56)$ were found to be non-hypertensive, of whom $n=3$ weaned dose and $n=13$ stopped antihypertensive medication whilst HDBPM. 5.4\% $(n=3)$ were found to have uncontrolled hypertension. None of the children required admission to hospital to manage their hypertension.

Conclusions: HDBPM is acceptable to children and their families; we demonstrate its feasibility as an out-of-office measurement technique in young children and those in whom ABPM is not possible.

Disclosure: None

References:

1. Clothier JC, Rigby E, Sinha MD. Home blood pressure monitoring in infants and children. Arch Dis Child. 2012;97(8):704-8.

P-18 The importance of auditing ward blood pressure machines (BPMs) and cuffs

\section{Willy Choon Kon Yune ${ }^{* 1}$, S. Philip Lewis ${ }^{2}$}

${ }^{1}$ University of Manchester School of Medicine, Manchester, United Kingdom, ${ }^{2}$ Stockport NHS Foundation Trust, Stockport, United Kingdom

Introduction: There should be a manual BPM machine in every hospital inpatient ward [1] and all BPMs should be validated. Previous studies have demonstrated poor maintenance of BPMs and cuffs in this setting. We aimed to assess compliance with BIHS guidelines [2] in a large District General Hospital (DGH).

Methods: All manual and automated BPMs and cuffs were assessed in the medical, surgical and maternity wards of a large DGH. Each BPM was checked against the BIHS and dabl $^{\circledast}$ Educational Trust [3] lists of validated BP machines and for function, range of cuff sizes available, cuff attachments, state of Velcro ${ }^{\circledR}$, state of connecting tubes, inflation bulbs and valves and for the presence of any air pressure leaks.

Results: A total of $127 \mathrm{BPMs}$ were assessed. All the manual machines (Accoson Greenlight 300) were validated by the BIHS but none of the automated machines had BIHS or dabl ${ }^{\oplus}$ Educational validations. Of 99 machines on medical and surgical wards, $81 \%$ were automated and $19 \%$ manual. On maternity wards, $64 \%$ were manual and $36 \%$ automated. Two or more cuffs of different sizes were available on $41 \%$ of the 37 manual BP monitors and on $62 \%$ of the 90 automated BP machines. Faults were present in 9 out of 37 manual BP monitors (missing connectors 2, defective inflation valves 4 , faulty bulbs 3 ) and in 12 out of 90 automated BP monitors (not working despite battery being charged 4 , no cuff attachments 6 , not working 4 , Velcro ${ }^{\circledast}$ not holding 2).

Conclusions: All manual machines but none of the automated machines were validated. There was an inadequate range of cuffs available. Maintenance of BPMs, their cuffs and attachments was unsatisfactory. Guidelines for purchase and provision of BPMs with an appropriate range of cuffs as well as for their routine maintenance are required [4].

Disclosure: None

\section{References:}

1. MHRA Device bulletin DB2006 (03), Blood pressure measurement devices. London, UK: Medicine and Healthcare Products Regulatory Agency, UK Department of Health; 2006.

2. British and Irish Hypertension Society. For specialist use-British and Irish Hypertension Society I Registered UK Charity No. 287635. British and Irish Hypertension Society; 2019. https://bihsoc.org/bpmonitors/for-specialist-use/ Accessed 31 Mar 2019. 
3. Dableducational.org. dabl Educational Trust-information on validated blood pressure devices and monitors. 2019. http://www.dableducational.org Accessed 31 Mar 2019.

4. Carney SL, Gillies AH, Green SL, Paterson O, Taylor MS, Smith AJ. Hospital blood pressure measurement: Staff and device assessment. J Qual Clin Pract. 1999;19: 95-8.

\section{P-19 Omitted \\ P-20 Omitted}

P-21 Late effects of hypertensive disorders of pregnancy on central blood pressure, microcirculation and cardiovascular diseases: a cross-sectional case control study

Ayushi Singh ${ }^{* 1}$, Elisa Allievi', Mihari De Soysa ${ }^{1}$, Rhoda Cookey-Bresi ${ }^{1}$, Roberta Angheleanu ${ }^{1}$, Tarek Antonios ${ }^{1}$

${ }^{1}$ St George's, University of London, London, United Kingdom

Introduction: The risk of cardiovascular disease (CVD) increases in women with a history of hypertensive disorders of pregnancy (HDP). Although the mechanisms exploring this association are poorly understood, a reduction in skin capillary density or capillary rarefaction (CR), has been proposed in its pathogenesis. CR precedes the onset of pre-eclampsia (PE) and essential hypertension. Hence, this study aimed to investigate whether CR persists post HDP and therefore, acts as a significant risk factor for the consequent development of CVD.

Methods: A total of 86 non-pregnant women were studied (mean age $37 \pm 6$ years). Of these, 44 had a previous history of PE (mean age $37 \pm 9$ years), 29 had history of gestational hypertension (GH) (mean age $37 \pm 6$ years) and 13 were healthy controls (mean age $35 \pm 5$ years). We measured central systolic blood pressure (cSBP), aortic augmentation index (AIx), pulse pressure (PP) and capillary density on the dorsum of the middle finger using noninvasive intra-vital capillaroscopy.

Results: GH group had higher cSBP (128.93 \pm $10.3 \mathrm{mmHg}$ vs $111.15 \pm 11.02 \mathrm{mmHg}, p<0.0001)$, SBP $(124.66 \pm 8.7 \mathrm{mmHg}$ vs $109.81 \pm 11.1 \mathrm{mmHg}, p=0.001)$ DBP $(76.33 \pm 9.0 \mathrm{mmHg}$ vs $67.8 \pm 8.4 \mathrm{mmHg}, p=0.025)$ and $\mathrm{PP}(48.3 \pm 8.6 \mathrm{mmHg}$ vs $41.9 \pm 4.5 \mathrm{mmHg}, p=0.031)$, compared to controls. GH also had a higher SBP (mean difference $7.5 \mathrm{mmHg}, 95 \%$ CI 0.84 to $14.23 \mathrm{mmHg}, p=$ 0.022 ) and $\mathrm{PP}$ (mean difference $6.26 \mathrm{mmHg}, 95 \% \mathrm{CI} 2.01$ to $10.51 \mathrm{mmHg}, p=0.002$ ) compared to PE. Moreover, PE group had higher cSBP (mean difference $11.63 \mathrm{mmHg}$,
95\%CI 1.76 to $21.50 \mathrm{mmHg}, p=0.015)$ compared to controls. No significant differences were seen in AIx or capillary density between groups.

Conclusions: Women with a history of GH are more likely to have systemic arterial stiffness, as a result of raised cSBP, PP and SBP, compared to women with history of PE and healthy controls. Further larger studies are needed to characterise the role of microcirculation in the causation of long-term increased risk of CVD in women with a history HDP.

Disclosure: None

P-22 Importance of non-obstructive coronary artery disease and blood pressure in determining stress myocardial blood flow in asymptomatic people with type 2 diabetes

Gaurav Gulsin ${ }^{* 1}$, Lavanya Athithan ${ }^{1}$, Kelly Parke ${ }^{1}$, Joanne Wormleighton ${ }^{2}$, Peter Kellman ${ }^{3}$, Aparna Deshpande $^{2}$, Gerry McCann ${ }^{1}$

${ }^{1}$ University of Leicester, Leicester, United Kingdom, ${ }^{2}$ University Hospitals of Leicester NHS Trust, Leicester, United Kingdom, ${ }^{3}$ National Heart, Lung, and Blood Institute, Bethesda, MD, USA

Introduction: The relationship between coronary artery calcium score (CAC), a marker of epicardial coronary artery disease, blood pressure (BP) and hyperaemic myocardial blood flow in asymptomatic people with type 2 diabetes (T2D) and no prior history of cardiovascular disease is unknown.

Methods: We prospectively enrolled adults with T2D and healthy controls, with no history of cardiovascular disease. Subjects underwent bio-anthropometric profiling, 24h ambulatory BP monitoring, CT CAC and adenosine stress cardiac MRI with automated inline myocardial blood flow quantification [1]. Subjects with reversible perfusion defects were excluded.

Results: A total of 40 people with T2D (age $65 \pm 7$ years, BMI $\left.31 \pm 6 \mathrm{~kg} / \mathrm{m}^{2}, \mathrm{HbA} 1 \mathrm{c} 7.2 \pm 0.9 \%\right)$ and 11 healthy controls (age $59 \pm 8$ years, BMI $26 \pm 3 \mathrm{~kg} / \mathrm{m}^{2}$, HbAlc $5.6 \pm$ $0.3 \%$ ) were included. Mean $24 \mathrm{~h}$ systolic blood pressure was higher in people with T2D than controls $(127 \pm 9$ vs $115 \pm 8 \mathrm{mmHg}$, respectively, $p=0.007$ ) but there was no difference in mean $24 \mathrm{~h}$ diastolic blood pressure $(p=0.782)$. Coronary artery atherosclerotic burden was higher in T2Ds compared with controls (total CAC $310 \pm 569$ vs $86 \pm 175$, respectively, $p=0.043$ ). Global stress myocardial blood flow was lower in T2Ds than in controls $(1.7 \pm 4.7$ vs $2.0 \pm$ $3.8 \mathrm{~mL} / \mathrm{g} / \mathrm{min}$, respectively, $p=0.045$ ), but there was no 
difference in resting myocardial blood flow $(p=0.629)$ between groups.

In those with T2D, total CAC was the only univariable predictor of global stress myocardial blood flow, with which it showed an inverse correlation $(r=-0.396, p=0.015)$. In a multivariable model containing age, gender, mean $24 \mathrm{~h}$ systolic blood pressure and CAC, CAC was the only independent predictor of global stress myocardial blood flow $(\beta=-0.373, p=0.045)$.

Conclusions: In asymptomatic people with T2D and no reversible perfusion defects, CAC but not blood pressure is an independent determinant of stress myocardial blood flow. Non-obstructive epicardial coronary disease should therefore be accounted for when assessing the potential causes of microvascular dysfunction in these patients.

Disclosure: None

\section{References:}

1. Kellman P, Hansen MS, Nielles-Vallespin S, Nickander J, Themudo R, Ugander M, et al. Myocardial perfusion cardiovascular magnetic resonance: optimized dual sequence and reconstruction for quantification. J Cardiovasc Magn Reson. 2017;19(1):43.

\section{P-23 Is hyperuricaemia associated with hypertension in psoriatic arthritis?}

\section{Celine Esuruoso $^{* 2}$, Bernie Colaco ${ }^{1}$, Abdul-majeed Salmasi $^{1,2}$}

${ }^{1}$ Central Middlesex Hospital, Park Royal, United Kingdom, ${ }^{2}$ Imperial College, School of Medicine, United Kingdom

Introduction: The association between hyperuricaemia and hypertension has been reported. However, previous studies demonstrated no association between hyperuricaemia and hypertension in patients with psoriasis. This current study aims to determine whether there is an association between hyperuricaemia and hypertension in a cohort of patients with psoriatic arthritis, a condition in which hyperuricaemia is a comorbidity.

Methods: The medical notes of 73 patients aged 25-88 years (24 males and 57 females) with psoriatic arthritis at the Central Middlesex Hospital were checked for hyperuricaemia and hypertension. The subjects were 40 Caucasians, 27 Indo-Asians and 6 Afro-Caribbean. Patients were considered hypertensive if they were on anti-hypertensive medication or had elevated blood pressure recordings. A chi-squared statistical test was conducted using IBM's SPSS software.

Results: Of the 73 patients, 9 had hyperuricaemia. Proportionally, $50 \%$ of the hyperuricaemic patients $(n=6)$ were hypertensive. $19 \%$ of patients with hypertension had hyperuricaemia compared to $7 \%$ of the normotensive. Despite that chi-squared test was non-significant $(p=014)$. There was no gender or ethnic differences in the results.

Conclusions: In this pilot study, there is no association between hypertension and hyperuricaemia in psoriatic arthritis patients. This is similar to the previously published results showing no association between hyperuricaemia and hypertension in patients with psoriasis. There was no gender or ethnic preferences in the results. Further studies with a larger sample size are suggested.

Disclosure: None

P-24 A study of Factors AssoCiated with Treatment Resistant HYpertension (FACT-RHY): description of phenotypical and biochemical characteristics of patients with true treatment-resistant hypertension compared to treated patients with hypertension

Mohammed Hameed ${ }^{* 1,2}$, Lesley Horton ${ }^{2}$, Sayeed Haque ${ }^{1}$, Paramjit Gill $^{3}$, Indranil Dasgupta ${ }^{1,2}$

${ }^{1}$ University of Birmingham, Birmingham, United Kingdom, ${ }^{2}$ Birmingham Heartlands Hospital, Birmingham, United Kingdom, ${ }^{3}$ University of Warwick, Warwick, United Kingdom

Introduction: Treatment-resistant hypertension (TRH) is present in around 10 percent of the patients with treated hypertension. Its prevalence is often overestimated, a consequence of failure to exclude non-adherence and white coat hypertension. We aim to describe phenotypical and biochemical characteristics of TRH in the first 100 patients recruited to FACT-RHY study (a study of Factors AssoCiated with Treatment Resistant HYpertension).

Methods: Patients aged 18-80 years with treated hypertension and without any confirmed causes of secondary hypertension were eligible. All patients gave written consent to attend the study visit where detailed clinical history, examination, biochemical and physiological testing was performed. Patients with uncontrolled blood pressure (average BpTRU $>140 / 90$ ) taking $\geq 3$ antihypertensives or if BP controlled on $\geq 4$ agents and in both case at least 3 antihypertensives must be detectable by urine antihypertensive assay. All other patients were defined as a control group.

Results: Forty-one patients had TRH, 53 were females and 51 Caucasians. Patients with TRH were older, had significantly higher waist-hip and waist-height ratios, a longer duration of hypertension and, higher prevalence of left ventricular hypertrophy and diabetes mellitus compared to controls. Patients with TRH were prescribed a higher number of antihypertensives and other medications 
including diuretics and lipid-lowering therapy. Biochemical testing showed that a lower estimated glomerular filtration rate and serum album and a higher kappa-lambda ratio, NTproBNP, high-sensitivity Troponin I and aldosterone-renin ratio were associated with TRH. There were no differences in urinary albuminuria, 24-h urinary catecholeamines and metadrenalines and daily salt intake estimated from 24-h urinary sodium excretion. The 10-year Qrisk2 was significantly higher at $21.0 \%$ in TRH compared to $7.6 \%$ in controls.

Conclusions: Patients with TRH have significantly higher comorbidities and medication burden culminating in a higher prevalence of target-organ damage and consequently a higher future risk of cardiovascular and cerebrovascular disease.

Disclosure: None

P-25 Evaluation of vascular endothelial dysfunction through measurement of serological biomarkers and reactive hyperaemia index in patients with true-treatment resistant hypertension

Mohammed Hameed $^{* 1,2}$, Sarah Hopkins ${ }^{3}$, Shakil Ahmed , $^{3}$ Sayeed Haque ${ }^{1}$, Asif Ahmed ${ }^{3}$, Paramjit Gill, Indranil Dasgupta ${ }^{1,2}$

${ }^{1}$ University of Birmingham, Birmingham, United Kingdom, ${ }^{2}$ Birmingham Heartlands Hospital, Birmingham, United Kingdom, ${ }^{3}$ Aston University, Birmingham, United Kingdom, ${ }^{4}$ University of Warwick, Birmingham, United Kingdom

Introduction: Association between hypertension and endothelial dysfunction is well-established. Studies have shown the presence of a higher degree of endothelial dysfunction in treatment-resistant hypertension (TRH). We aim to assess and compare endothelial dysfunction, by measuring levels of vascular biomarkers and reactive hyperaemia index (RHI), in patients with true TRH and non-TRH controls in the first 100 patients recruited to FACT-RHY study (a study of Factors AssoCiated with Treatment Resistant HYpertension).

Methods: Peripheral arterial tone (PAT) was measured pre and post complete brachial arterial occlusion for $5 \mathrm{~min}$ using EndoPAT. The RHI is the post-to-pre occlusion PAT signal ratio in the occluded arm, relative to the same ratio in the control arm, corrected for baseline vascular tone of the occluded arm. Enzyme-linked immunosorbent assay was used to measure levels of sFlt-1, ICAM-1, VCAM-1, endothelin-1, and endoglin. Patients with adherenceconfirmed TRH (either uncontrolled BP on $\geq 3$ agents or controlled BP on $\geq 4$ agents) were compared against a control group.

Results: Forty-three patients underwent EndoPAT assessment and biomarkers were measured in all patients. There was no significant difference in the proportion of patients with abnormal RHI in patients with TRH compared to controls; $7(41.2 \%)$ and $8(30.7 \%)$ respectively $(p=$ 0.528). Median (interquartile range) serum endothelin-1 concentration was significantly higher in the TRH group compared to control group; $1.98(1.61-2.38) \mathrm{pg} / \mathrm{mL}$ and $1.81(1.40-2.26) \mathrm{pg} / \mathrm{mL}(p=0.001)$. Median concentration of VCAM-1 was higher in the TRH group at 1136.9 (1024.0-1229.7) $\mathrm{ng} / \mathrm{mL}$ compared to the control group at $1049.0(956.3-1131.6) \mathrm{ng} / \mathrm{mL}(p=0.017)$. There was no significant difference in serum concentrations of sFLT-1, endoglin, and ICAM-1 in the two groups. No significant correlations were present between RHI and the biomarkers.

Conclusions: Serum endothelin-1 and VCAM-1 were found to be higher suggesting higher level of endothelial dysfunction in patients with TRH.

Disclosure: None

P-26 Assessment of fluid excess and body composition using bioimpedance spectroscopy in patients with adherence-proven treatment-resistant hypertension

\section{Mohammed Hameed $^{* 1,2}$, Sayeed Haque ${ }^{1}$, Paramjit Gill ${ }^{3}$, Indranil Dasgupta ${ }^{1,2}$}

${ }^{1}$ University of Birmingham, Birmingham, United Kingdom, ${ }^{2}$ Birmingham Heartlands Hospital, Birmingham, United Kingdom, ${ }^{3}$ University of Warwick, Warwick, United Kingdom

Introduction: Fluid retention has been shown to be a predominant factor in the development of treatment-resistant hypertension (TRH) and has been successfully shown to be an important therapeutic target with diuretics such as aldosterone receptor antagonists. We aim to assess fluid excess and body composition using bioimpedance spectroscopy in the first 100 patients recruited to FACT-RHY study (a study of Factors AssoCiated with Treatment Resistant HYpertension).

Methods: All patients with treated hypertension without any confirmed causes of secondary hypertension underwent bioimpedance spectroscopy using the Fresenius Body Composition Monitor (BCM), which has been validated against gold standard reference methods to assess fluid excess in patients with end-stage renal disease. Patients with adherence-confirmed TRH (either uncontrolled BP on $\geq 3$ agents or controlled BP on $\geq 4$ agents) were compared against a non-TRH control group of patients. 
Results: Forty-one patients with TRH and 59 patients with treated hypertension underwent bioimpedance spectroscopy. The mean absolute fluid excess, in litres, was higher in the TRH compared to controls but did not reach statistical significance. The BCM identified only 5 patients with absolute fluid excess (>1.1L) overall, 3 of whom had TRH. Interestingly, however $47 \%$ were found to be underhydrated $(<-1.1 \mathrm{~L})$ and the remaining $48 \%$ normally hydrated. Another measure of hydration, extracellular water to intracellular water ratio, was higher in the TRH group compared to controls; $0.86(0.09)$ and $0.82(0.07)$ respectively $(p=0.013)$. Fluid excess correlated negatively with serum albumin and measured daily salt intake, and positively with aldosterone-renin ratio and urine albumincreatinine ratio. Lean tissue index and fat tissue index did not differ significantly in the two study groups.

Conclusions: Although some measures suggest fluid excess was higher, generally more patients were found to be underhydrated which may be a consequence of high diuretic use in the TRH group.

Disclosure: None

P-27 Comparison of carotid-to-femoral pulse wave velocity as a maker of arterial stiffness in patients with true treatment-resistant hypertension and non-resistant treated patients with hypertension

\section{Mohammed Hameed $^{* 1,2}$, Sayeed Haque ${ }^{1}$, Charles Ferro ${ }^{3}$, Paramjit Gill $^{4}$, Indranil Dasgupta ${ }^{1,2}$}

${ }^{1}$ University of Birmingham, Birmingham, United Kingdom, ${ }^{2}$ Birmingham Heartlands Hospital, Birmingham, United Kingdom, ${ }^{3}$ University Hospitals Birmingham NHS Foundation Trust, Birmingham, United Kingdom, ${ }^{4}$ University of Warwick, Warwick, United Kingdom

Introduction: Aortic stiffness as quantified by carotid-tofemoral pulse wave velocity (cfPWV) is considered a gold standard marker of asymptomatic organ damage in hypertension. There is no clear evidence to suggest that patients with treatment-resistant hypertension (TRH) have an increased PWV compared to non-TRH patients with studies reporting both higher and no difference in PWV. We aim to compare the cfPWV in TRH and non-TRH controls in the first 100 patients recruited to FACT-RHY study (a study of Factors AssoCiated with Treatment Resistant HYpertension).

Methods: All patients with treated hypertension without any confirmed causes of secondary hypertension underwent pulse wave analysis and cfPWV measurements using the Vicorder system. Patients with TRH (either uncontrolled $\mathrm{BP}$ on $\geq 3$ agents or controlled $\mathrm{BP}$ on $\geq 4$ agents) were compared against a non-TRH control group of patients.
Adherence to antihypertensives was confirmed using urine antihypertensive assay.

Results: Both central and peripheral pulse pressures were significantly higher in the patients with TRH compared to controls. Augmentation index, either alone or adjusted to a heart rate of 75 beats per minute, was not found be significantly different in the two groups of patients. Mean cfPWV was significantly higher in patients with TRH when compared to controls; $10.0 \pm 2.2 \mathrm{~m} / \mathrm{s}$ and $8.8 \pm 2.1 \mathrm{~m} / \mathrm{s}$ respectively $(p=0.008)$. Univariate correlations of study parameters with cfPWV showed a strong positive correlation between increasing age and cfPWV $(r=0.527 p<0.001)$. Gender, ethnicity, BMI and smoking status had no significant correlation with cfPWV, whilst smoking pack years, longer duration of hypertension, presence of diabetes mellitus, peripheral systolic BP and mean arterial pressure had significant positive correlation with cfPWV.

Conclusions: Patients with TRH were found to have a higher cfPWV with a relatively strong positive correlation with age and peripheral systolic BP.

Disclosure: None

P-28 Evaluation of patients' beliefs and perceptions of their antihypertensive medications and their association with adherence and presence of treatment-resistant hypertension using beliefs about medicines questionnaire

\section{Mohammed Hameed ${ }^{* 1,2}$, Sayeed Haque ${ }^{1}$, Paramjit Gill ${ }^{3}$, Indranil Dasgupta ${ }^{1,2}$}

${ }^{1}$ University of Birmingham, Birmingham, United Kingdom, ${ }^{2}$ Birmingham Heartlands Hospital, Birmingham, United Kingdom, ${ }^{3}$ University of Warwick, Warwick, United Kingdom

Introduction: Non-adherence to antihypertensive medications is common in patients with treatment-resistant hypertension (TRH) and is present in up to $50 \%$, the reasons for which are multifactorial. We aim to assess patients' beliefs about medications and their association with adherence status and TRH in first 100 patients recruited to the FACT-RHY (a study of Factors AssoCiated with Treatment-Resistant HYpertension) study using the beliefs about medicines questionnaire (BMQ). The BMQ has been showed to be associated with adherence in numerous chronic illnesses.

Methods: The BMQ comprises 18 statements, divided into 4 subscales, each is rated on a five-point Likert scale. The scores from the Likert scale (strongly-agree, agree, uncertain, disagree, strongly-disagree) are aggregated for each subscale with the highest score indicating stronger beliefs. Specificnecessity and specific-concern subscales assess patients' 
beliefs about the necessity of and their concerns about the potential adverse-effects of taking antihypertensive medications. General-overuse and general-harm subscales evaluate patients' views about how doctors use medications and the extent to which they perceive medications as harmful.

Results: All 100 patients attending the study visit completed the BMQ. Sixty patients were found to be adherent to $80 \%$ of the antihypertensives prescribed that were detectable on urine antihypertensive assay. The Chronbach's alpha for specific-necessity, specific-concerns, generaloveruse and general-harm subscales were $0.83,0.71,0.77$ and 0.68 respectively. In unadjusted analysis, none of the 4 subscales were associated with adherence status. In logistic regression analysis general-overuse and generalharm subscales were independently associated with nonadherence. Mean scores for specific-necessity, specificconcerns and general-overuse subscales were significantly higher in TRH patients compared to controls.

Conclusions: Our study suggests that patients with TRH believe that their medicines are necessary which outweigh their concerns about taking medicines. Furthermore, patients' perceptions of medicines as being harmful and their overuse by doctors was associated with nonadherence.

Disclosure: None

P-29 Leptin is associated with serum uric acid level and its excretion in a sample of adult men

Lanfranco D'elia* ${ }^{*}$, Alfonso Giaquinto ${ }^{I}$, Francesco Cappuccio $^{2}$, Roberto Iacone ${ }^{I}$, Ornella Russo ${ }^{I}$, Pasquale Strazzullo $^{1}$, Ferruccio Galletti ${ }^{1}$

${ }^{1}$ Federico II University of Naples, Naples, Italy, ${ }^{2}$ Warwick Medical School, University of Warwick, Coventry, UK

Introduction: Leptin (LPT) is associated with cardiovascular risk factors (e.g. hypertension, kidney disease, insulin resistance and excess body weight). Experimental studies showed that LPT might affect serum uric acid (SUA), by modulation of the SUA excretion. However, there are few observational data on the relationship between LPT and SUA in general population. Therefore, the aim of the present study was to evaluate the relationship between LPT and SUA and its excretion in a large middle-aged male general population.

Methods: A sample of 930 adult male individuals (mean age: 52 years) without therapy for high SUA was included in the analysis (the Olivetti Heart Study).

Results: SUA was significantly and positively associated with LPT, BMI, waist circumference, blood pressure, insulin resistance and $\mathrm{C}$-reactive protein $(p<0.01)$, while inversely with renal function $(p=0.01)$. The multivariate analysis confirmed the association between LPT and SUA after adjustment for potential confounders $(p<0.01)$. This trend was also detected for normal weight and excess body weight participants, separately. Moreover, LPT was inversely associated with excretion of SUA $(p<0.01)$, also in multivariate analysis $(p=0.03)$.

Conclusions: The results of this study indicate a positive association between circulating LPT levels and SUA, independently of main potential confounders, both in normal weigh and excess body weight men. Furthermore, these findings suggest an inverse relationship between LPT and SUA excretion.

Disclosure: All authors declare that they have no conflict of interest.

P-30 Assessing population dietary intake of sodium, potassium and iodine in adults in middle-income countries: a case study from the Republic of Moldova

Lanfranco D'Elia', Galina Obreja ${ }^{5}$, Angela Ciobanu ${ }^{4}$, Joao Breda ${ }^{3}$, Jo Jewell', Francesco Cappuccio ${ }^{* 1,6}$

${ }^{1}$ University of Warwick, Coventry, United Kingdom, ${ }^{2}$ Regional Office for Europe, Copenhagen, Denmark, ${ }^{3}$ European Office for Prevention and Control of NCDs, Moscow, Russian Federation, ${ }^{4}$ Country Office of Moldova, Chisinau, Moldova, ${ }^{5}$ National Centre for Public Health, Chisinau, Moldova, ${ }^{6} \mathrm{UHCW}$ NHS Trust, Coventry, United Kingdom

Introduction: High sodium $(\mathrm{Na})$ and inadequate potassium (K) intake are important determinants of high blood pressure (BP) and cardiovascular diseases (CVDs), as well as iodine deficiency is a public health problem. CVDs are the leading cause of morbidity and mortality in the Republic of Moldova. There is no reliable measure of $\mathrm{Na}, \mathrm{K}$ and iodine intake in Moldova. The aim of the present survey was to establish current baseline average consumption of $\mathrm{Na}, \mathrm{K}$ and iodine in a random sample of men and women in Moldova.

Methods: The survey sample was randomly selected by the National Bureau of Statistics to be nationally representative. Participants attended a screening including demographic, anthropometric and physical measurements by standardized methods. Dietary Na (salt), K and iodine intakes were assessed by $24 \mathrm{~h}$ urinary $\mathrm{Na}$ (UNa), K (UK) and iodine (UI) excretions. Urinary creatinine was measured to evaluate the completeness of the urine collection.

Results: Eight hundred and fifty-eight (326 men, 18-69 years) were included in the analysis (response rate $66 \%)$. Mean $\mathrm{UNa}$ was $173( \pm 79 \mathrm{SD}) \mathrm{mmol} / \mathrm{day}$, equivalent to $10.8 \mathrm{~g}$ of salt/day, and $\mathrm{K}$ excretion 73 $( \pm 31) \mathrm{mmol} /$ day, equivalent to $3.7 \mathrm{~g} /$ day. Only $11 \% \mathrm{had}$ 
a salt intake below the WHO recommended target of $5 \mathrm{~g} /$ day, and $50 \%$ ate enough $\mathrm{K}(>90 \mathrm{mmol} /$ day $)$. Mean UI was $225( \pm 152) \mathrm{mcg} / 24 \mathrm{~h}, 41 \%$ had adequate iodine intake, whilst $29 \%$ were still deficient and $30 \%$ had excessive intake. The prevalence of hypertension (BP $>140 / 90 \mathrm{mmHg}$ or on anti-hypertensive medications) was $45.5 \%$, with no difference between sexes. Twothirds of responders $(74.3 \%)$ were not taking medications and had raised BP, with higher prevalence in men $(82.4 \%)$ compared to women $(69.2 \%)$.

Conclusions: There is need for intensive awareness campaigns and health promotion to improve the take up of preventive strategies aiming at reducing salt consumption and increasing potassium and iodine intake in the Republic of Moldova. Furthermore, the level of untreated hypertension is still high, particularly in men.

Disclosure: None

P-31 Predicting adverse responses to physical activity in young adults with suboptimal blood pressure

Odaro J Huckstep ${ }^{1,2}$, Maryam Alsharqi ${ }^{* 1,3}$, Wilby Williamson $^{I}$, Winok Lapidaire ${ }^{I}$, Fernando Telles ${ }^{1}$, Ashley Verburg $^{1}$, Jennie Han ${ }^{1}$, Charlotte Herdman ${ }^{1}$, Linda Arnold $^{I}$, Afifah Mohamed ${ }^{1}$, Henry Boardman ${ }^{1}$, Helen Dawes $^{4}$, Charlie Foster ${ }^{5}$, Adam J Lewandowski ${ }^{1}$, Paul Leeson ${ }^{1}$

${ }^{1}$ University of Oxford, Oxford, United Kingdom, ${ }^{2}$ United States Air Force Academy, CO, USA, ${ }^{3}$ Imam Abdulrahman Bin Faisal University, Dammam, Saudi Arabia, ${ }^{4}$ Oxford Brookes University, Oxford, United Kingdom, ${ }^{5}$ University of Bristol, Bristol, United Kingdom

Introduction: In a population, greater physical activity is associated with lower blood pressure (BP) but, for an individual, significant heterogeneity in experience and BP response to exercise is observed [1]. We hypothesised certain cardiovascular phenotypes associated with suboptimal BP may result in adverse responses to acute and/or chronic exercise. Our objective is to perform multi-modality deepphenotyping in a cohort of young adults with a range of BP to investigate variation in cardiac response to exercise and identify baseline predictors of response to exercise interventions.

Methods: 146 young adults ( $27 \pm 5$ years) with a range of BP were recruited between 2011 and 2016 to undergo cardiac, vascular and brain magnetic resonance imaging, lifestyle and psychological assessment (including 7 day accelerometery) and blood sampling. A sub-group $(n=71)$ underwent cardiopulmonary exercise testing with stress echocardiography to assess the myocardial response to exercise at $40 \%, 60 \%$, and $80 \%$ of peak exercise load as well as biochemical response to exercise (e.g. plasma copeptin release).

Results: Participants with suboptimal BP $(128.8 \pm 9.5 /$ $76.7 \pm 7.98 \mathrm{mmHg}$ ) showed higher left ventricular mass indexed to end-diastolic volume $(P=0.007)$ and lower longitudinal systolic strain when compared with optimal BP participants $(109.7 \pm 6.46 / 66.4 \pm 5.65)$. During exercise the suboptimal group had a smaller increase in ejection fraction up to $40 \%$ workload $(P<0.001)$ (Fig. 1) and achieved ejection fraction at $40 \%$ workload inversely associated with the post-exercise physiological stress marker, plasma copeptin $\left(r^{2}=0.115, P=0.043\right)$. The suboptimal BP group reported greater exertion on exercise and lower enjoyment of physical activity.

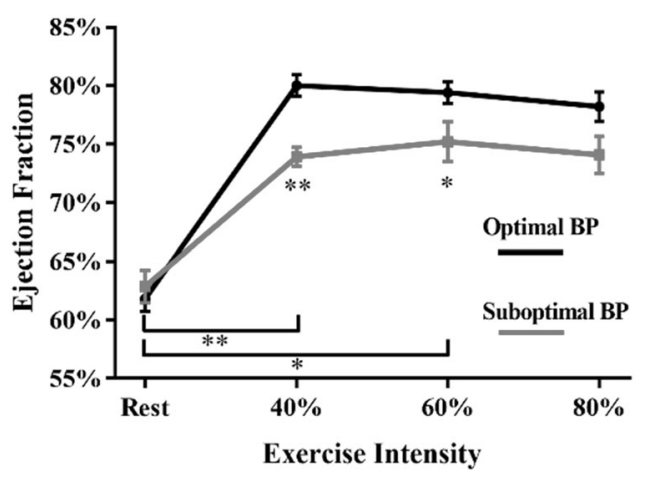

Conclusions: Response and experience of exercise varies in young adults according to levels of BP. Further work will identify specific baseline phenotypes including left atrial haemodynamics and right ventricular function that predict this response and whether interventions can mitigate the impact in clinical exercise interventions.

Disclosure: None

References:

1. Williamson W, Foster C, Reid H, et al. Will exercise advice be sufficient for treatment of young adults with prehypertension and hypertension? A systematic review and meta-analysis. Hypertension. 2016;68 (1):78-87.

\section{P-32 (Abstract Withdrawn)}

P-33 Evidence of endothelial dysfunction in young South Asian women during reactive and exercise hyperaemia: does higher BMI contribute?

Isra Khan ${ }^{* 1}$, Sophie Richardson ${ }^{* 1}$, Abimbola Aiku, Janice Marshall 
${ }^{1}$ University of Birmingham, Birmingham, United Kingdom.

${ }^{2}$ University of Ibadan, Nigeria

Introduction: Prevalence of cardiovascular disease (CVD) including hypertension is higher in South Asians (SAs) than White Europeans (WEs). This has been associated with endothelial dysfunction, diabetes and obesity ${ }^{1}$. Whether endothelial dysfunction is present in young SA women and whether vasodilator prostaglandins might protect them is unknown.

Methods: Arterial blood pressure (ABP), forearm blood flow (FBF), forearm vascular conductance (FVC: FBF/ $\mathrm{ABP}$ ) were recorded in $8 \mathrm{SA}$ and $8 \mathrm{WE}$ women (student volunteers; $20 \pm 0.5 ; 20 \pm 0.3$ years) at rest and following $2 \mathrm{~min}$ arterial occlusion (reactive hyperaemia) and $2 \mathrm{~min}$ rhythmic contractions at 30 and $60 \%$ maximum voluntary contraction (MVC) after placebo and after cyclooxygenase inhibition (aspirin $600 \mathrm{mg}$ p.o).

Results: In SAs vs WEs, resting ABP was $120 \pm 4.3 / 73 \pm$ 3.6 vs $114 \pm 3.1 / 73 \pm 2.7 \mathrm{mmHg}$, BMI: $26 \pm 2.2$ vs $22 \pm 0.9$, physical activity: $1900 \pm 530$ vs $3100 \pm 490$ MET min/week, parental hypertension: 4/8; 1/8. Peak FVC in reactive hyperaemia was $0.4 \pm 0.02$ and $0.56 \pm 0.5$ conductance units (CU) in SAs vs WEs $(P=0.07)$, but exercise hyperaemia at $30 \% \mathrm{MVC} 0.36 \pm 0.02$ vs $0.45 \pm 0.03 * \mathrm{CU}$ and at $60 \% \mathrm{MVC}$ was $0.48 \pm 0.05$ vs $0.54 \pm 0.09 * \mathrm{CU}$ were blunted in SAs (*: SA vs WE, $P<0.05)$. In WEs, COX inhibition attenuated reactive hyperaemia (to $0.43 \pm 0.56^{\S} \mathrm{CU},{ }^{\S}$ : aspirin vs placebo) and exercise hyperaemia at $30 \%$ MVC $(0.32 \pm$ $\left.0.46^{\S} \mathrm{CU}\right)$ and $60 \%\left(0.43 \pm 0.09^{\S}\right)$, but in SAs, only peak hypertaemia at $30 \% \mathrm{MVC}$ was attenuated $\left(0.25 \pm 0.03^{8} \mathrm{CU}\right.$; reactive hyperaemia: $0.38 \pm 0.02$, $60 \% \mathrm{MVC}: 0.37 \pm 2.1 \mathrm{CU}$ ). Since $5 / 8 \mathrm{SAs}$ and $2 / 8 \mathrm{WEs}$ had $\mathrm{BMI}>25 \mathrm{~kg} / \mathrm{m}^{2}$, women were also split by BMI irrespective of ethnicity. In high BMI women, $\mathrm{COX}$ inhibition attenuated exercise hyperaemia at $60 \%$ MVC, but had no effect on reactive hyperaemia or exercise hyperaemia at $30 \% \mathrm{MVC}$, whereas in normal BMI women, $\mathrm{COX}$ inhibition attenuated reactive and exercise hyperaemia at 30 and $60 \%$ MVC.

Conclusions: In young SA women, endotheliumdependent reactive and exercise hyperaemia are blunted with a depressed contribution of vasodilator prostaglandins. This may be attributed to their higher BMI and predispose them to CVD.

Disclosure: None

References:

1. Gupta M, Singh N, Verma S, Circulation. 2006;113:924-29.

P-34 Body size and blood pressure: an unfair focus on fat?
Shikai Yu ${ }^{* 1}$, Kathleen Connolly ${ }^{I}$, Jessica Middlemiss ${ }^{I}$, Jean Woodcock-Smith ${ }^{1}$, Ian Wilkinson ${ }^{I}$, Carmel McEniery ${ }^{I}$

${ }^{1}$ University of Cambridge, Cambridge, United Kingdom

Introduction: The relationship between body size and blood pressure (BP) is well recognized. Body size also associates with cardiac output (CO) — the key haemodynamic determinant of BP in young adults. However, it is unclear whether fat mass or lean mass primarily associates with BP and its determinants, since lean mass also increases in obesity. The aim of this study was to examine the relationship between elements of body composition, BP and its haemodynamic determinants in young adults.

Methods: In-depth phenotyping was undertaken in 271 healthy individuals (153 males; $28 \pm 6$ years) across a range of body size and BP levels. Body composition was assessed using electrical bioimpedance; $\mathrm{CO}$ and stroke volume (SV) using inert gas rebreathing. Peripheral vascular resistance (PVR) was calculated from $\mathrm{CO}$ and mean arterial pressure (MAP). All analyses were conducted separately for males and females.

Results: Fat free mass (FFM) ranged from $38.9-87.4 \mathrm{~kg}$ (males) and $35.5-59.3 \mathrm{~kg}$ (females). Fat mass (FM) ranged from $2.9-44.3 \mathrm{~kg}$ (males) and 6.1-72.2 kg (females). Multiple linear regression analyses revealed that FFM was significantly and positively associated with systolic $\mathrm{BP}\left(\beta^{\text {male }}=0.39 ; \beta^{\text {female }}\right.$ $=0.76 ; \mathrm{mmHg}), \mathrm{CO}\left(\beta^{\text {male }}=0.09 ; \beta^{\text {female }}=0.17 ; \mathrm{L} / \mathrm{min}\right)$ and $\mathrm{SV}\left(\beta^{\text {male }}=1.71 ; \beta^{\text {female }}=2.89 ; \mathrm{ml}\right)$, and negatively with PVR $\left(\beta^{\text {male }}=-12.37 ; \quad \beta^{\text {female }}=-27.91 ; \quad\right.$ dynes $\left./ \mathrm{s} / \mathrm{cm}^{5}\right), \quad$ in both sexes. FM was significantly associated with diastolic BP $\left(\beta^{\text {male }}=0.28 ; \beta^{\text {female }}=0.32 ; \mathrm{mmHg}\right)$ and $\mathrm{MAP}\left(\beta^{\text {male }}=0.31\right.$; $\left.\beta^{\text {female }}=0.34 ; \mathrm{mmHg}\right)$ in both sexes, and with PVR $(\beta=8.17$; dynes $\left./ \mathrm{s} / \mathrm{cm}^{5}\right)$ and heart rate $(\beta=0.71$; beats $/ \mathrm{min})$ in females. Differences between sexes in systolic BP, diastolic BP, MAP, $\mathrm{CO}, \mathrm{SV}$ and PVR were eliminated by matching for FFM, but not FM.

Conclusions: FFM is the dominant determinant of systolic BP in young adults, via increased $\mathrm{CO}$ and $\mathrm{SV}$ and reduced PVR. However, FM is the dominant determinant of diastolic BP and MAP. The impact of lean mass on BP elevation should not be overlooked in young adults.

Disclosure: None

P-35 The association between obesity and arterial stiffness from adolescence through to emerging adulthood and the confounding role of blood pressure

Chloe Park $^{* 1}$, Laura Howe ${ }^{2}$, Alicja Rapala ${ }^{1}$, Debbie Lawlor $^{2}$, George Davey-Smith ${ }^{2}$, Marietta Charakida ${ }^{1}$, 
Frida Dangardt ${ }^{I}$, John Deanfield ${ }^{I}$, Nish Chaturvedi, Alun Hughes ${ }^{1}$

${ }^{1}$ UCL Institute for Cardiovascular Science, London, United Kingdom, ${ }^{2}$ MRC Integrative Epidemiology Unit, University of Bristol, Bristol, United Kingdom

Introduction: Adult obesity is associated with greater arterial stiffness however there is controversy regarding the association in children, with some evidence of an inverse association between body mass index (BMI) and pulse wave velocity (PWV) during childhood. We investigated this relationship over the period of emerging adulthood (17-24 years).

Methods: 388517 year olds (2152 female) had measurements of carotid-femoral PWV, anthropometry and blood pressure (BP). Measurements were repeated aged $24.0 \pm 0.8$ years $(n=1659)$. Linear regression and linear mixed models were used to assess sex-stratified associations between BMI and PWV at both ages and age interactions.

Results: From 17 to 24 years BMI increased by +2.4 $[2.2-2.6] \mathrm{kg} / \mathrm{m}^{2}(p<0.0001)$ in males and $+2.0[1.8,2.2]$ $\mathrm{kg} / \mathrm{m}^{2}(p<0.0001)$ in females while PWV increased by $+0.65[0.56,0.74] \mathrm{m} / \mathrm{s}$; (mean $[95 \% \mathrm{CI}] ; p<0.0001)$ in males and by $+0.55[0.49,0.61] \mathrm{m} / \mathrm{s}(p<0.0001)$ in females.

BMI showed no convincing association with $\mathrm{PWV}$ in males at 17 years $(\beta[\mathrm{CI}]:-0.001[-0.018,0.016] \mathrm{m} / \mathrm{s}$, $p=0.9) \quad$ or 24 years $(\beta[\mathrm{CI}]:-0.002[-0.025,0.021]$, $p=0.9$ ). A weak positive association was observed in females at $17 \mathrm{yrs}(\beta[\mathrm{CI}]: 0.010[-0.0007,0.02], p=0.07)$ which was stronger at 24 years $(\beta[C I]: 0.028[0.015,0.040]$, $p<0.0001$ ), although evidence of modification by age in females was equivocal $\left(p_{\text {interaction }}=0.08\right)$.

After further adjustment for confounders (mean arterial pressure, socioeconomic status, education and smoking) an inverse association emerged in both males $(\beta[\mathrm{CI}]:-0.023$ $[-0.041,-0.0053], p=0.02)$ and females $(\beta[\mathrm{CI}]:-0.014$ $[-0.025,-0.004], p=0.01)$ aged 17 years. At 24 years there was no convincing association in males $(\beta[\mathrm{CI}]:-0.012$ $[-0.040,0.015], \quad p=0.4) \quad$ or females $\quad(\beta[\mathrm{CI}]: 0.008$ $[-0.006,0.023], p=0.2)$.

Conclusions: In adolescence and emerging adulthood BMI is positively associated with PWV only in females. Moreover, this association is explained by confounding by BP. In adolescence once BP is accounted for higher BMI is associated with lower PWV in both sexes; however PWV rises with age and the inverse association between BMI and PWV is not seen in young adults. Emerging adulthood may be an important time to control BMI and provide long term cardiovascular benefits.

Disclosure: None
P-36 Risk of MACE events and arterial stiffness in the Whitehall II study

Valencia-Hernández C.A. ${ }^{* 1}$, McEniery C. $^{2}$, Wilkinson I.B. ${ }^{2}$, Ahmadi-Abhari S. ${ }^{1}$, Shipley M.J. , Brunner E.J. ${ }^{1}$

${ }^{1}$ Research Department of Epidemiology and Public Health, University College London, UK, ${ }^{2}$ Division of Experimental Medicine and Immunotherapeutics, University of Cambridge, Cambridge, UK

Introduction: Arterial stiffness is a predictor of cardiovascular risk. Previously published literature showed that the risk of cardiac events is $40 \%$ higher per each additional standard deviation of carotid-femoral pulse wave velocity (PWV). No additional studies assessing the predictive value of repeated measurements have been published up to date.

Methods: The Whitehall II study is an on-going cohort of civil servants followed every 4-5 years. Data used for this analysis consists of two measurements of pulse wave velocity (PWV) by 4 years taken during phases 9 (2008-2009) and 11 (2012-2013), from 4998 participants (3680 men; 1318 women). Data from death certificates and Hospital Episode Statistics was used to extract information regarding fatal and non-fatal stroke, myocardial infarction and coronary heart disease. Using this information, a composite outcome index known as Major Adverse Cardiovascular Events was built. The follow-up period started at the phase 9 clinic and finished in March 2017.

Results: Empty models showed a hazard ratio of 1.3 (1.1-1.2) per each metre per second of carotid-femoral PWV. The final model adjusted for sociodemographic covariates, comorbidities and health behaviours showed a HR of 1.1 (95\%CI: $1.1-1.2 p<0.001)$. When predicting non-cardiovascular disease mortality, the empty model showed a Hazard Ratio of 1.07, but the fully-adjusted model was not statistically significant. PWV predicted all-cause mortality HR 1.09 (95\%CI: 1.04-1.13 $p<0.05$ ). A Cox model using two PWV measurements did not find an association with MACE events (HR 1.03 95\%CI: 0.96-1.09 $p: 0.345)$. The predictive power of models using two measurements of pulse wave velocity (C-statistic: 0.7361 ROC: 0.5410) was not higher than those of models using a single measurement (C-statistic: 0.7388 ROC: 0.6155).

Conclusions: Predictive power of multiple measurements of PWV was not superior compared to a single measurement in participants of the Whitehall II study.

Disclosure: None

\section{References:}

1. Ben-Shlomo Y, Spears M, Boustred C, et al. Aortic pulse wave velocity improves cardiovascular event prediction: an individual participant meta-analysis of 
prospective observational data from 17,635 subjects. J Am Coll Cardiol. 2014;63(7):636-46. https://doi.org/ 10.1016/j.jacc.2013.09.063.

2. Marmot M, Brunner E. Cohort Profile: The Whitehall II study. Int J Epidemiol. 2005;34(2):251-6. https:// doi.org/10.1093/ije/dyh372.

3. Britton A, Milne B, Butler T, et al. Validating selfreported strokes in a longitudinal UK cohort study (Whitehall II): Extracting information from hospital medical records versus the Hospital Episode Statistics database. BMC Med Res Methodol. 2012;12(1):83. https://doi.org/10.1186/1471-2288-12-83.

\section{P-37 Impact of inter-arm blood pressure difference on cardiovascular risk estimation in primary care}

\section{Sinead McDonagh ${ }^{* 1}$, Ben Norris ${ }^{2}$, A. Jayne Fordham ${ }^{3}$, Maria Greenwood ${ }^{3}$, Suzanne Richards ${ }^{4}$, John Campbell, Christopher Clark ${ }^{1}$}

${ }^{1}$ University of Exeter, Exeter, United Kingdom, ${ }^{2}$ Royal Devon and Exeter NHS Foundation Trust, Exeter, United Kingdom, ${ }^{3}$ Mid Devon Medical Practice, Witheridge Medical Centre, Exeter, United Kingdom, ${ }^{4}$ University of Leeds, Leeds, United Kingdom

Introduction: The INTERPRESS-IPD Collaboration demonstrated that a systolic inter-arm difference in blood pressure (IAD) is an independent risk marker for cardiovascular events after adjustment for QRISK2, Framingham or ASCVD scores. This approach offers improved risk prediction through reclassification of individuals across risk categories and can inform initiation of treatment for primary prevention of cardiovascular disease in those close to current pharmaceutical intervention thresholds. The aim of this study was to model this approach of adjusting cardiovascular risk prediction scores for IAD in a primary care population.

Methods: Individuals aged 45-75 years, free of cardiovascular disease, had bilateral blood pressure measured three times, simultaneously, during National Health Service (NHS) Health Checks in one general practice in Devon, England. QRISK2 scores were calculated as part of the Health Check process; we subsequently calculated Framingham and ASCVD scores during analysis. The risk scores were adjusted by taking account of IAD using hazard ratios derived from our INTERPRESS-IPD analyses.

Results: Complete data existed for 334 participants [mean (standard deviation); age: 57.4 (9.3), systolic/diastolic blood pressure: 132 (14)/79 (8.5)]. Mean QRISK2, Framingham and ASCVD scores were 8.0 (6.9), 10.7 (8.1) and 6.9 (6.5), respectively before adjustment for IAD and 8.9 (7.7), 11.2 (8.5) and 7.1 (6.7) afterwards. Overall, 18 individuals were reclassified from below to above either the $10 \%$ or $20 \%$ QRISK2 risk thresholds, and more individuals were reclassified across the corresponding Framingham (10) and ASCVD (3) thresholds.

Conclusions: Our findings confirm that systolic IADs can be applied to refine cardiovascular risk estimates in a UK primary care population. By taking account of systolic IAD, individual decisions on interventions for primary prevention of cardiovascular disease can be personalised and could facilitate targeting of treatment to those at greater than average cardiovascular risk.

Disclosure: None

References: Clark CE, et al. J Hypertens. 2018;36:105.

\section{P-38 (Abstract Withdrawn)}

P-39 The rural dispensing practice: does it achieve better medication adherence and clinical outcomes compared to non-dispensing practices? A crosssectional analysis of routine data

\section{Mayam Gomez-Camo ${ }^{1}$, John Campbell, Christopher Clark $^{*}$, Bianca Wiering ${ }^{l}$, Gary Abel ${ }^{I}$}

${ }^{1}$ University of Exeter, Exeter, United Kingdom

Introduction: When general practices dispense medications, as opposed to issuing prescriptions which may, this removes one cause of non-adherence to medication. The implications of this difference are unknown; we hypothesised that dispensing may achieve better medication adherence than prescribing alone. This could impact clinical outcome measures dependent on good medication adherence such as control of hypertension.

Methods: We analysed Quality and Outcome Framework $(\mathrm{QOF})^{1}$ data from 2016/17 linked to NHS Business Services Authority dispensing status for England. QOF performance indicators were classified as:

(i) indicators dependent on medication adherence (e.g. percentage of hypertensive patients with blood pressure (BP) $\leq 150 / 90 \mathrm{mmHg}$ ); (ii) indicators dependent on prescribing but not on adherence (e.g. prescribing antiplatelet drugs in ischaemic heat disease) or (iii) indicators unrelated to prescribing or adherence (e.g. assessment of stroke risk in atrial fibrillation). Using mixed-effects logistic regression we estimated differences between dispensing and nondispensing practices, with adjustment for percentage of practice population aged $\geq 65$, sex, practice deprivationlist size, single-handed status and rurality. 
Results: Data existed for practices; (1\%). Dispensing practices had more patients aged $\geq 65$, lower deprivation, were more often single-handed rural. Seven of nine QOF indicators dependant on adherence were better achieved in dispensing than non-dispensing practices, including greater achievement of target hypertension (Odds ratio 1.07; 95\% CI 1.04-1.11, $p<$ 0.0001 ). In contrast, we only found differential achievement for one of nine indicators related to prescribing but not adherence, and one of six indicators unrelated to prescribing or adherence.

Conclusions: Between 3\% and 10\% of prescriptions go unfilled by pharmacies. These findings offer initial evidence that dispensing practices may achieve better outcomes than prescribing practices through improved medication adherence. Further work is required to clarify the possible underlying mechanisms for these observations.

Disclosure: None

\section{References:}

1. Quality and Outcomes Framework (QOF)—2016-17. https://digital.nhs.uk/data-and-information/publications/sta tistical/quality-and-outcomes-framework-achievementprevalence-and-exceptions-data/quality-and-outcomes-fra mework-qof-2016-17

P-40 Antihypertensive drug concordance in patients with apparent resistant hypertension: a tertiary hypertension referral centre experience

\section{Mohammed Meher Ali Chaudhry ${ }^{* 1}$, Tarek Francis Antonios $^{\text {I }}$}

${ }^{1}$ St George's, University of London, London, United Kingdom

Introduction: Individuals with resistant hypertension $(\mathrm{RH})$ have a 50\% greater risk of cardiovascular disease events compared to those with well-controlled hypertension [1]. Nonadherence to antihypertensive medication is a major cause of $\mathrm{RH}$, with rates reported as high as $66 \%$ [2]. The aim of this study was to assess the prevalence of non-adherence in individuals with apparent $\mathrm{RH}$ referred to the Blood Pressure Unit at St George's University Hospital NHS foundation Trust.

Methods: After the optimisation of drug regimens and the exclusion of white coat and secondary hypertension, drug adherence was assessed using liquid chromatography-mass spectrometry analysis of urine samples in 124 individuals who met the criteria for RH. All included subjects confirmed adherence to their prescribed antihypertensive medication on several occasions, including when urine samples were obtained.

Results: Non-adherence was found in 71 individuals $(57.3 \%)$. Of these, $45(63.4 \%)$ had partial non-adherence and $26(36.6 \%)$ had complete non-adherence. Non-adherent individuals were significantly younger, had a shorter history of hypertension, more likely to be a female (OR: 3.07 ; $95 \%$ CI: $1.45-6.47 ; p=0.003$ ) and have depression (OR: 11.66; 95\% CI 1.47-92.19; $p=0.004$ ), and less likely to have concomitant cardiovascular disease and/or target organ damage (OR: 0.42 95\% CI 0.19-0.92; $p=0.028$ ), type 2 diabetes mellitus (OR: 0.40 ; $95 \%$ CI: $0.19-0.83 ; p=0.013$ ), chronic kidney disease (OR: $0.34 ; 95 \%$ CI $0.12-0.92 ; p=0.028$ ) and retinopathy (OR: $0.14 ; 95 \%$ CI $0.04-0.51 ; p=0.001$ ). Low levels of adherence were observed more in individuals on diuretics.

Conclusions: In individuals with apparent RH, poor adherence to antihypertensive therapy is very common and was observed in $57 \%$ of individuals despite their insistence of treatment adherence in the preceding days. The assessment of adherence at an early stage in individuals with apparent RH is essential for tailoring treatment strategies and saving vital healthcare resources.

Disclosure: None

\section{References:}

1. Daugherty SL, Powers JD, Magid DJ, et al. Incidence and prognosis of resistant hypertension in hypertensive patients. Circulation.2012;358:1635-42. https:// doi.org/10.1161/CIRCULATIONAHA.111.068064. pmid: 22379110.

2. Hyman D, Pavlik V. Medication adherence and resistant hypertension. J Hum Hypert. 2015;29:213-18.

\section{P-41 Optimal strategies for blood pressure lowering treatment}

Emily Herrett $^{* 1}$, Sarah Gadd ${ }^{1}$, Rod Jackson ${ }^{2}$, Krishnan Bhaskaran $^{1}$, Elizabeth Williamson ${ }^{1,3}$, Tjeerd van Staa ${ }^{4,5}$, Reecha Sofat ${ }^{6}$, Adam Timmis ${ }^{7}$, Liam Smeeth ${ }^{1}$

${ }^{1}$ London School of Hygiene and Tropical Medicine, London, United Kingdom, ${ }^{2}$ University of Auckland, Auckland, New Zealand, ${ }^{3}$ HDR UK, London, United Kingdom, ${ }^{4}$ University of Manchester, Manchester, United Kingdom, ${ }^{5}$ Utrecht Institute of Pharmaceutical Sciences, Utrecht, The Netherlands, ${ }^{6}$ University College London, London, United Kingdom, ${ }^{7}$ Queen Mary University London, London, United Kingdom

Introduction: The current approach to blood pressure treatment is guided predominantly by blood pressure thresholds. This is despite strong evidence that the benefits of blood pressure reduction are observed across the blood pressure spectrum and that absolute treatment benefits are proportional to pre-treatment absolute cardiovascular risk. The aim of this study was to investigate the implications of alternative strategies for offering blood pressure treatment in the UK.

Methods: This is a cohort study using data from 1.2 million UK primary care patients without cardiovascular disease 
(CVD), followed from 2011 for a median 4.3 years in the Clinical Practice Research Datalink, linked to Hospital Episode Statistics and Office for National Statistics mortality data. The strategies compared were (i) UK National Institute for Health and Care Excellence (NICE) 2019 guidelines, (ii) using blood pressure alone $(140 / 90 \mathrm{mmHg}$ threshold), (iii) using predicted cardiovascular risk (QRISK2 $\geq 10 \%$ ten-year cardiovascular risk). For each strategy, we estimated the proportion of patients eligible for treatment and the number of CVD events that could be prevented with treatment.

Results: 327,428 individuals (26.8\% of the cohort) were eligible for treatment under the 2019 NICE guideline, 481,859 $(39.4 \%$ ) based on blood pressure $\geq 140 / 90 \mathrm{mmHg}$, and 357,840 (29.3\%) based on QRISK2 $\geq 10 \%$. The CVD event rates among those eligible for each strategy were 14.9, 11.6 and 17.1 per 1000 person years, respectively. Scaled to the UK population, we estimated that 270,232 events would be avoided under the NICE strategy (29 patients treated for ten years per event avoided), 301,523 avoided using blood pressure only (38 treated/event avoided); and 322,921 avoided using QRISK2 (27 treated/event avoided).

Conclusions: A cardiovascular risk-based strategy could prevent over one fifth more CVD events than current guidance, with similar or better efficiency in terms of number treated per event avoided.

Disclosure: Funded by NIHR PDF-2016-09-029, Wellcome/Royal Society (107731/Z/15/Z)

Data were obtained from the CPRD. CPRD is a research service that provides primary care and linked data for public health research. CPRD data governance, and our own license to use CPRD data, do not allow us to distribute or make available patient data directly to other parties. Researchers may apply for data access at www.cprd.com, and must have their study protocol approved by the Independent Scientific Advisory Committee for MHRA database research (details at www.cprd.com/isac).

\section{P-42 Impact of the hypertension guidelines in prescrip- tion trend of thiazide diuretics in the UK}

\section{Luca Faconti, Ryan McNally ${ }^{*}$, Franca Morselli, Bushra Farukh, Philip J. Chowienczyk ${ }^{1}$ \\ ${ }^{1}$ King's College London, London, United Kingdom}

Introduction: Since the publication of the most recent NICE Hypertension guidelines (2011) [1] diuretics are not regarded as first line treatment in the UK and thiazide-like molecules, such as Chlortalidone (CLTD) and Indapamide (INDA) rather than thiazide-type (Hydrochlorothiazide (HCTZ) and Bendroflumethiazide (BDTZ)) are the preferred option in patients who are initiated on diuretic treatment. Here we examine the prescription trend in thiazide diuretics after the implementation of the guidelines.

Methods: Prescription Cost Analysis data was gathered for Northern Ireland (2017) from the Business Services Organisation [2], Scotland (2015/16) from the Information Services Division [3], England (2017) from the Prescribing and Medicines Team NHS Digital and Wales (2017) [4] from NHS Wales Shared Services Partnership [5] as well as data from 2010 for all four regions. Both generic and branded medicines containing BDTZ, HCTZ, CLTD and INDA were included provided they were licensed for hypertension as per the British National Formulary. The total quantity of all dispensed items was calculated, and each individual diuretic was expressed as a percentage of the total. The datasets can be made available upon request to the principal investigator.

Results: Overall the prescriptions of thiazide diuretics have declined over the years (from 1071,154,641 in 2010 to $742,292,346$ in 2016/2017). Despite some geographical differences, in 2016/2017 prescriptions of thiazide-type overrided thiazide-like (74.3\% of the total) and BDTZ was the most commonly prescribed diuretic in the UK $(68.8 \%$ of the total). The use of INDA has increased since 2010 (from $7.3 \%$ to $24.3 \%$ ) while the one of CTLD represented only $2.5 \%$ in 2010 and $1.3 \%$ in 2016/2017. Between the four UK regions, Scotland has been least affected by the change in prescription trend (increase of $4.3 \%$ in thiazidelike vs. $15.8 \%$ overall the UK).

Conclusions: Thiazide-type diuretics (in particularly BDTZ) are still the most commonly prescribed diuretics in the UK while the use of CLTD is limited.

Disclosure: None

\section{References:}

1. NICE. Hypertension in adults: diagnosis and management (CG127). NICE; 2011. https://www.nice.org.uk/ guidance/cg127. Accessed 12 Feb 2019

2. http://www.hscbusiness.hscni.net/services/1806.htm

3. https://www.isdscotland.org/Health-Topics/ Prescribing-and-Medicines/Community-Dispensing/ Prescription-Cost-Analysis/

4. https://digital.nhs.uk/data-and-information/publica tions/statistical/prescription-cost-analysis

5. https://gov.wales/statistics-and-research/prescriptionsdispensed-community/?lang $=\mathrm{en}$

P-43 Using routine care data to investigate the comparative effectiveness of first line anti-hypertensive drugs according to age, ethnicity and diabetes strata 
Sarah-Jo Sinnott ${ }^{1}$, Ian Douglas ${ }^{1}$, Elizabeth Williamson ${ }^{1}$, Liam Smeeth ${ }^{1}$, Laurie Tomlinson ${ }^{* 1}$

${ }^{1}$ London School of Hygiene and Tropical Medicine, London, United Kingdom

Introduction: NICE clinical guidelines for hypertension are currently being updated. Recommendations for firstline anti-hypertensive drugs have in the past been based on clinical studies and physiological understanding, but comparative effectiveness in UK routine care is unknown.

Methods: We conducted a new-user, propensity-score matched cohort study using UK primary care data from the Clinical Practice Research Datalink 2007-2017, linked to Hospital Episode Statistics. We investigated change in systolic (sBP) and diastolic blood pressure (dBP) at 12, 26 and 52 weeks post-initiation separately for CCB vs ACEI/ $\mathrm{ARB}$, and thiazides vs CCB. To investigate the NICE treatment algorithm we used mixed linear models with interactions to model the effect of the drug class across:

(1) Age: $<55 />55 y e a r s$ in a non-black, non-diabetic population.

(2) Ethnicity: non-black/black in a non-diabetic population.

(3) Diabetes: present/absent in whole population.

Results for dBP and weeks 26 and 52 not shown given space constraints.

Results: We identified 87,440 initiators of ACEI/ARB, 67,274 of CCB and 22,040 of thiazides. In the baseline population, $64.6 \%$ were $\geq 55$ years, $2.5 \%$ black and $11.5 \%$ diabetic. At 12 weeks:

1. Among non-black people without diabetes, $\mathrm{CCB}$ achieved larger reductions in SBP than ACEI/ARB and this did not differ by age: $<55$ years$0.93 \mathrm{mmHg}\left(95 \% \mathrm{CI}-1.65 \_:-0.22\right), \quad 255$ years $-1.02 \mathrm{mmHg}\left(95 \% \mathrm{CI}_{-}-1.57 \_:-0.47\right)$.

2. Among people without diabetes, $\mathrm{CCB}$ achieved larger reductions in sBP than $\mathrm{ACE} / \mathrm{ARB}$ and this differed by ethnicity: black $-4.97 \mathrm{mmHg}\left(95 \% \mathrm{CI}_{-}-8.56 \_\right.$:-1.38 ), non-black $-0.96 \mathrm{mmHg}\left(95 \% \mathrm{CI} \_-1.42 \_\right.$:-0.49 ).

3. In the whole population, $\mathrm{CCB}$ achieved larger reductions in SBP than ACEI/ARB and this differed by diabetic status: non-diabetic $-1.01 \mathrm{mmHg} \_(95 \%$ CI_-1.48_:_-0.54),

diabetic $-0.54 \mathrm{mmHg}\left(95 \% \mathrm{CI}-1.65 \_: 0.58\right)$.

Comparing thiazide vs $\mathrm{CCB}, \mathrm{CCB}$ achieved larger reductions in sBP with no evidence of differences across subgroups.
Conclusions: Routine care data can be used to examine anti-hypertensive effectiveness. Our results show that NICE anti-hypertensive class recommendations result in larger BP reductions for black people but not according to age or diabetic status.

\section{Disclosure: None}

\section{References:}

1. Herrett, E., Gallagher, A. M., Bhaskaran, K., Forbes, H., Mathur, R., van Staa, T., \& Smeeth, L. (2015). Data resource profile: clinical practice research datalink (CPRD). International journal of epidemiology, 44(3), 827-836.

P-44 Bendroflumethiazide versus indapamide for primary hypertension: observational (BISON) study within CPRD

Tatiana V. Macfarlane ${ }^{* 1}$, Amy Rogers ${ }^{I}$, Robert W.V. Flynn $^{1}$, Steven V. Morant ${ }^{1}$, Isla S. Mackenzie ${ }^{1}$, Thomas M. MacDonald

${ }^{1}$ University of Dundee, Dundee, United Kingdom

Introduction: The 2011 NICE Guideline recommend a thiazide-like diuretic in preference to a thiazide diuretic when initiated or changed [1]. Bendroflumethiazide and indapamide are the dominant drugs of this class prescribed in UK [2-4]. A systematic review of indapamide (IND) vs. bendroflumethiazide (BFZ) for hypertension [5] found a lack of studies and no evidence of superiority of IND v BFZ.

The aim of this observational study was to compare cardiovascular outcomes in patients prescribed indapamide versus bendroflumethiazide, as the first-line diuretic in the treatment of hypertension.

Methods: Clinical Practice Research Datalink (CPRD) was used to identify patients with first diagnosis of hypertension after 1987 who were registered with "up-to-standard" practices for at least 12 months. We excluded patients with prior outcome of interest, cancer diagnosis or other prescriptions for antihypertensives.

Information was extracted on socio-economic status, risk factors, co-morbidities and prescribed medication. Two groups of patients with incident prescriptions for indapamide or bendroflumethiazide were formed. The primary outcome was a per-protocol composite of nonfatal myocardial infarction, acute coronary syndrome, stroke, acute decompensated heart failure or death from cardiovascular causes. Cox regression was used for statistical analysis. 
Results: There were 5747 patients in the indapamide group, and 22,988 propensity score matched patients in the bendroflumethiazide group. In the per-protocol analysis of primary outcome there was no statistically significant difference between the groups (HR $0.8695 \%$ CI $0.68,1.09$ ).

Conclusions: This study showed no difference in the primary analysis between BFZ and IND. However, confounding and sample size issues suggest the need for a randomised study directly comparing the drugs. One such trial, EVIDENCE (Evaluating Diuretics in Normal Care), is currently in pilot [6].

Disclosure: TM was funded by Farr UK.

\section{References:}

1. National Institute for Health and Clinical Excellence (NICE). Hypertension in adults: diagnosis and management. Clinical guideline August 2011. https://www.nice.org.uk/guidance/cg 127

2. http://www.isdscotland.org/Health-Topics/

Prescribing-and-Medicines/Publications/2016-08-16/ opendata.asp

3. OpenPrescribing.net, EBM DataLab, University of Oxford, 2017.

4. https://www.cprd.com/

5. Macfarlane TV, Pigazzani F, Flynn RWV, MacDonald TM. The effect of indapamide vs. bendroflumethiazide for primary hypertension: a systematic review. Br J Clin Pharmacol. 2019;85 (2):285-303.

6. Flynn RWV, Rogers A, Flynn A, MacDonald TM, Mackenzie IS, Doney A. Can cluster randomisation of prescribing policy be used to efficiently generate drug safety and effectiveness data? Pilot phase of the evidence study. Pharmacoepidemiol Drug Saf. 2018;27:3-521.

\section{P-45 Effects of aspirin for prevention of vascular events in black patients (review)}

Nusirat Bello ${ }^{* 1}$, Anthony Waka ${ }^{2}$
${ }^{1}$ University of Ilorin, Ilorin, Nigeria, ${ }^{2}$ University of Benin, Benin, Nigeria

Introduction: The risk-benefit profile of aspirin remains debatable in prevention of atherosclero-thrombotic occlusive vascular complications, the major causes of global mortality. Hypertension is a significant risk factor highly prevalent in blacks. A review was carried out to assess the efficacy and safety of aspirin in primary and secondary prevention of vascular events in black patients.

Methods: Online published studies by November, 2018 were systematically searched in PubMed, Cochrane and Google Scholar databases since their inception using combination of the key words: 'black', 'Africa', 'aspirin', 'acetylsalicylic acid', 'vascular events', 'myocardial infarction (MI)', 'stroke', 'vascular death' or 'vascular mortality'. Efficacy end points were non-fatal and fatal MI, stroke and vascular deaths, while safety end points were bleeding and other adverse events reported.

Results: 12 studies (11 clinical trials and a review) were identified within the last 15 years (eight published five years ago), mainly conducted in western countries. 18,250 $(11.57 \%)$ of the 157,699 patients were blacks. One study had only black patients, otherwise patients were predominately white, and sub-group analyses of the end points were generally not reported. 9053 black patients (12.55\%) of the 113,609 patients took aspirin alone ranging from 25 $-1300 \mathrm{mg}$, or combination with an antithrombotic agent. Overall, aspirin showed reasonable efficacy in long term secondary prevention of stroke, MI and vascular death, and seemed better in preventing first MI than primary stroke. Serious bleeding increased with dose and primary prevention of stroke. Five other antithrombotic agents did not appear superior, severe and fatal bleeding and possibly vascular deaths increased in combination with aspirin.

Conclusions: Shortage in clinical trial evidence specific to black patients and broad geographical representation is concerning. Cautious use and monitoring of aspirin in black patients in clinical practice is recommended.

Disclosure: No conflicts of interest 